\title{
Tom70 mediates activation of interferon regulatory factor 3 on mitochondria
}

\author{
Xin-Yi Liu ${ }^{1, *}$, Bo Wei ${ }^{1, *}$, He-Xin Shi ${ }^{1}$, Yu-Fei Shan ${ }^{1}$, Chen Wang ${ }^{1}$ \\ ${ }^{1}$ Laboratory of Molecular Cell Biology, Institute of Biochemistry and Cell Biology, Shanghai Institutes for Biological Sciences, \\ Chinese Academy of Sciences, Shanghai 200031, China
}

Intracellular RNA viruses are sensed by receptors retinoic acid-inducible gene 1 (RIG-I)/melanoma differentiation-associated gene 5 (MDA5) that trigger the formation of MAVS signal complex on mitochondria. Consequently, this leads to the activation of TANK-binding kinase 1 (TBK1) and phosphorylation of interferon regulatory factor 3 (IRF3), both of which constitutively associate with cytosolic chaperone Hsp90. It remains largely unknown how MAVS activates TBK1/IRF3. In this study, we identified translocases of outer membrane 70 (Tom70), a mitochondrial import receptor, to interact with MAVS upon RNA virus infection. Ectopic expression or knockdown of Tom70 could enhance or impair IRF3-mediated gene expression, respectively. Mechanistically, the clamp domain (R192) of Tom70 interacts with the C-terminal motif (EEVD) of Hsp90, thus recruiting TBK1/IRF3 to mitochondria. Disruption of this interaction or mislocation of Tom70 sharply impairs activation of TBK1 and IRF3. Furthermore, host antiviral responses are significantly boosted or crippled in the presence or absence of Tom70. Collectively, our study characterizes Tom70 as a critical adaptor linking MAVS to TBK1/IRF3, revealing that mitochondrion is evolutionarily integrated with innate immunity.

Keywords: Tom70; MAVS; IRF3; Hsp90; antiviral innate immunity

Cell Research (2010) 20:994-1011. doi:10.1038/cr.2010.103; published online 13 July 2010

\section{Introduction}

Induction of type I interferons and pro-inflammatory cytokines represents the first line of host defense against viruses [1]. This antiviral response is initiated by germline-encoded pattern recognition receptors (PRRs). One of the PRR families is the Toll-like receptors (TLRs, including TLR3, TLR7-TLR8 and TLR9) that are predominantly expressed in the endosome of immune cells. Upon virus infection, these receptors detect the presence of viral nucleic acids in topologically extracellular compartment and recruit adaptor proteins (TRIF and MyD88), which ultimately activate transcription factors IRF3/IRF7 and $\mathrm{NF}-\mathrm{kB}[2]$. In contrast, retinoic acid-inducible gene 1 (RIG-I) and melanoma differentiation-associated gene

\footnotetext{
*These two authors contributed equally to this work. Correspondence: Chen Wang Tel: +86-21-54921185

E-mail: cwang01@sibs.ac.cn

Received 11 March 2010; revised 12 June 2010; accepted 12 June 2010; published online 13 July 2010
}

5 (MDA5) have recently been characterized as ubiquitous sensors for detecting cytosolic RNA viruses during primary responses of host cells [3-8]. Once RIG-I/MDA5 sense the viral RNAs, they trigger the formation of a signal complex on the mitochondrial outer membrane, which includes adaptor proteins MAVS/IPS1/VISA/Cardif, TRAF3 and TRADD [9-13]. This ultimately leads to the activation of TANK-binding kinase 1 (TBK1), which then phosphorylates interferon regulatory factor 3 (IRF3) on a series of Ser/Thr residues at its C-terminus [14-16]. In turn, IRF3 dimerizes, translocates into nucleus and recruits p300/CBP, which ultimately results in the early production of IFN- $\beta$ and subsequent establishment of antiviral state [17-19]. Several proteins have also been reported to regulate this signaling pathway. In addition, MAVS signal complex could activate IKK complex and $\mathrm{NF}-\kappa \mathrm{B}$.

Notably, MAVS per se localizes on the outer membrane of mitochondria through its C-terminal transmembrane (TM) domain [11]. In contrast, the rest of the reported components in the MAVS signal complex reside in the cytosol. Although TRAF3/TRADD are implicated 
to associate with MAVS, there is no direct interaction between MAVS and TBK1/IRF3 [20-22]. It remains largely unknown how MAVS relays the activation signal to TBK1/IRF3.

Although mitochondria encode some genetic information, almost all the mitochondrial proteins are synthesized in the cytosol and then transported into corresponding compartments in mitochondria. Recent breakthroughs on mitochondrial protein targeting demonstrate that translocases of outer membrane (Tom) complex is responsible for initial recognition of mitochondrial preproteins from the cytosol $[23,24]$. Tom70 and Tom 20 are characterized as major docking receptors to mediate the recognition by different mechanisms. Importantly, a subgroup of mitochondrial pre-proteins is constitutively associated with chaperone Hsp90, which is revealed to deliver this category of proteins to Tom70 $[25,26]$. In contrast, Tom 20 recognizes classical N-terminal signal peptides (positively charged amphipathic helices) found in most of the mitochondrial pre-proteins and this mechanism is Hsp90-independent [27]. Tom70 (608 aa) is anchored to the mitochondrial outer membrane via its Nterminal TM segments (1-60 aa). The remaining part of Tom70 is largely hydrophilic and exposed to the cytosol, which include a clamp domain (110-235 aa) and a core domain (250-608 aa). The clamp and core domains are implicated to mediate its interactions to Hsp90 and mitochondrial pre-proteins, respectively [25]. It is intriguing to address if Tom70 plays novel function(s) besides its role in the mitochondrial protein transport.

An unresolved issue is that why mitochondria is selected as the platform for intracellular antiviral signaling. Also, a critical missing link exists from MAVS to TBK1/IRF3. Therefore, we use proteomics approach to identify novel proteins in the MAVS signal complex. To our surprise, Tom70 is found to interact specifically with MAVS during RNA virus infection. In this study, we demonstrate that Tom70 plays an essential role in the antiviral response. Ectopic expression of Tom 70 potentiates IRF3-mediated gene expression. Conversely, depletion of Tom70 expression by RNA interference drastically decreases IFN- $\beta$ production, thus permitting viral infection and replication. Mechanistically, the clamp domain (R192) of Tom70 interacts with the C-terminal motif (EEVD) of Hsp90, thereby recruiting TBK1/IRF3 to mitochondria. Significantly, disruption of this interaction or mislocation of Tom70 sharply impairs the activation of TBK1 and IRF3. Taken together, these data indicate that Tom70 is a critical adaptor bridging TBK1/IRF3 to MAVS. The current study sheds new light on the evolutionary integration of mitochondria with antiviral innate immunity.

\section{Results}

Tom70 is a new component of MAVS signal complex on mitochondria

Given that cytosolic TBK1/IRF3 do not interact directly with mitochondrial MAVS, we reasoned that there might be unknown adaptor protein(s) on the outer membrane of mitochondria linking TBK1/IRF3 to MAVS during virus infection. To address this possibility, we expressed Flag-MAVS in HEK293T cells and then treated them with or without Sendai virus $(\mathrm{SeV})$ for $2 \mathrm{~h}$. After fractionation of the mitochondrial pellets, immunoprecipitation and silver staining of MAVS protein complex revealed a specific band $(\sim 80 \mathrm{kDa})$, the amount of which apparently increased upon $\mathrm{SeV}$ stimulation (Figure 1A). Mass spectrometry analysis unequivocally identified the translocase of outer membrane 70 (Tom70; 31 unique peptides covering $57.4 \%$ of the whole protein, data not shown), and immunoblotting of the same precipitates by Tom70 polyclonal antibody confirmed this observation (Figure 1B). Apparently, virus infection did not affect the expression of Tom70 (Figure 1C). Both confocal imaging and sub-cellular fractionation confirmed that Tom 70 and Tom 20 were exclusively expressed on mitochondria (Figures 1D and 4E). Immunoprecipitation assays revealed that Tom70 also associated with TRADD, TRAF6 and STING, but not with CARD9, suggesting that Tom70 is a novel component of the MAVS signalsome (Figure $1 \mathrm{E}$ and $1 \mathrm{~F})$ [12, 13, 30-32].

Exogenous expression of Tom70 potentiates IRF3-mediated gene expression

To explore the potential functional relevance of Tom70-MAVS interaction, we investigated whether Tom70 had any impact on IRF3 activation status. As shown in Supplementary information, Figure S1A and S1B, introduction of Tom 70 into HEK293 cells potentiated the induction of both IFN- $\beta$ - and PRDIII-I-luciferase reporters in a dose-dependent manner upon $\mathrm{SeV}$ infection or poly(I:C) stimulation. Also, Tom70 enhanced the expression of $\mathrm{kB}$-luciferase reporter under the same condition (Supplementary information, Figure S1A and S1B). As a control, overexpression of Tom 20 did not influence the IFN- $\beta$ - or PRDIII-I-luciferase reporters (Supplementary information, Figure S1C and S1D). Furthermore, we measured the induction of endogenous mRNAs from IRF3-responsive genes (including IFN- $\beta$, ISG56 and Rantes) by Q-PCR, after stimulating the cells by $\mathrm{SeV}$ infection or poly(I:C) transfection in the presence of exogenously expressed Tom70. Significantly, Tom70 potentiated the expression of endogenous IRF3-responsive genes (Figure 2A and 2B). In contrast, Tom20 had no ef- 


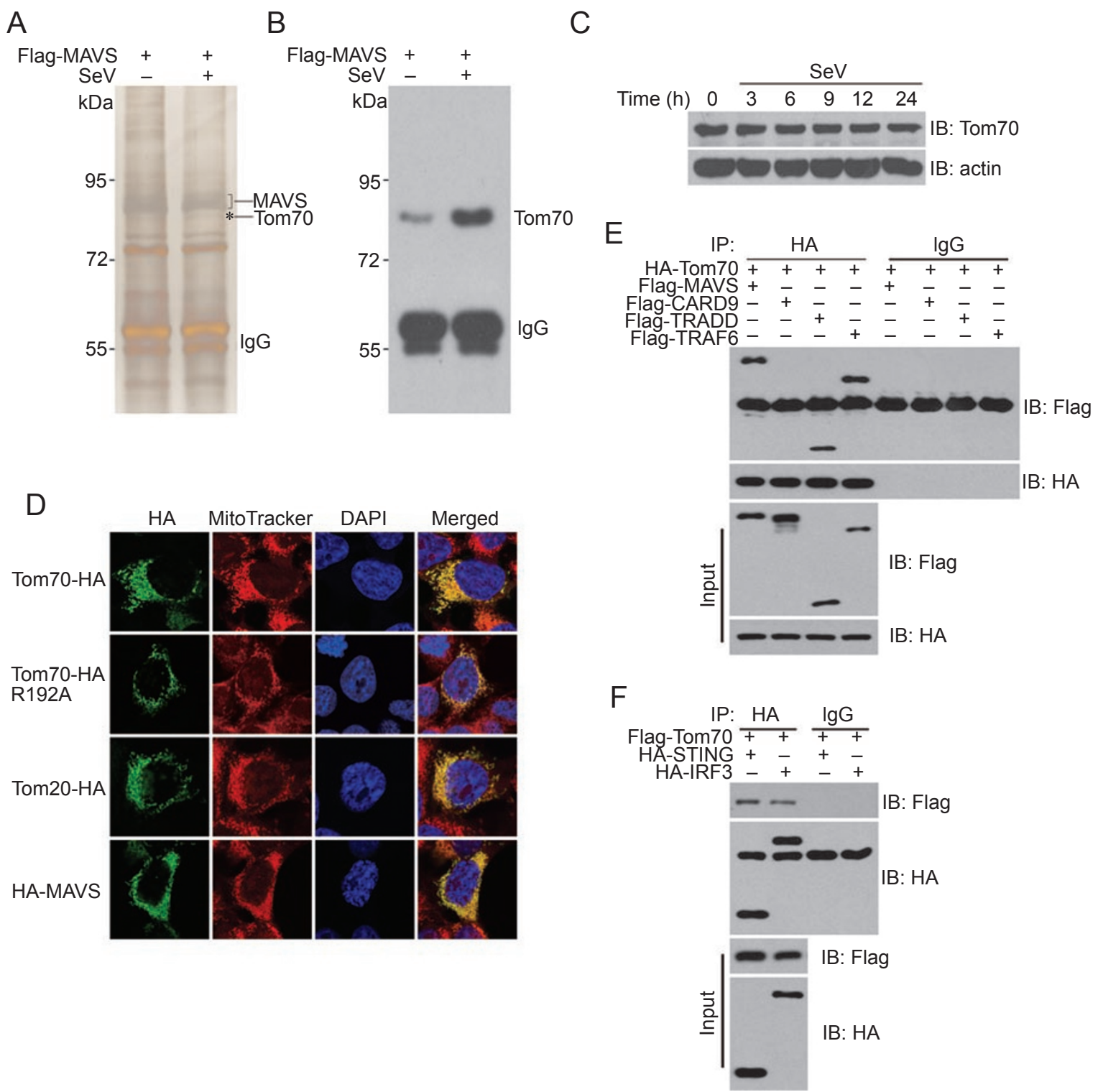

Figure 1 Identification of Tom70 as a new mitochondrial protein binding to MAVS. (A) HEK293T cells were transfected with Flag-MAVS and then stimulated with or without SeV for $2 \mathrm{~h}$. Cell lysates were subjected to immunoprecipitation with antiFlag monoclonal antibody. The immunoprecipitates were resolved in SDS-PAGE followed by silver staining. Specific band noted by asterisk was excised for MS identification. (B) The same samples from (A) were immunoblotted with anti-Tom70 polyclonal antibody. (C) HEK293 cells were stimulated with $\mathrm{SeV}$ for indicated time, and the cell lysates were immunoblotted with anti-Tom70 and anti- $\beta$-actin antibodies. (D) Tom70-HA, Tom70-HA(R192A), Tom20-HA and HA-MAVS were transfected into HEK293T cells individually, which were then stained with anti-HA antibody and imaged by confocal microscopy. The mitochondria were stained with MitoTracker. R192A, the point mutant of Tom70. (E, F) HEK293T cells were co-transfected with indicated constructs and then subjected to immunoprecipitation with control lgG or anti-HA antibody.

fect on the mRNA transcription of IRF3-regulated genes. In addition, ectopic expression of Tom 70 displayed no effect on TRIF-mediated activation of IFN- $\beta$-luciferase reporters or Wnt signaling (Supplementary information, Figure S1E and S1F).
To make it more physiologically relevant, we examined whether Tom70 regulated IRF3-induced gene expression in primary cells. Consistently, ectopic expression of Tom70 markedly potentiated the expression of endogenous IRF3-responsive genes both in BMDMs 
A

HEK293 cells
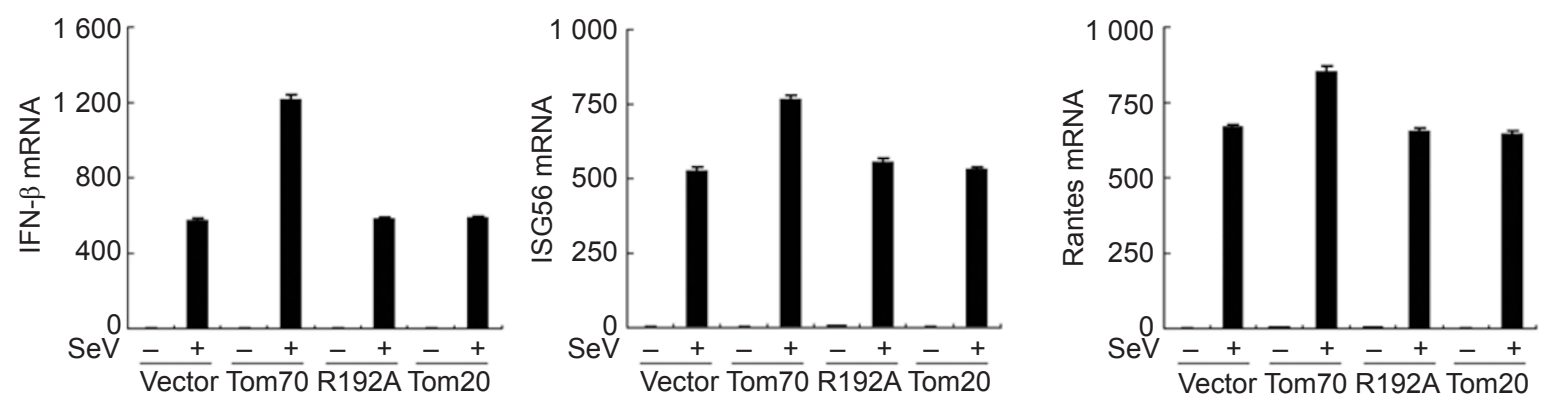

B

HEK293 cells
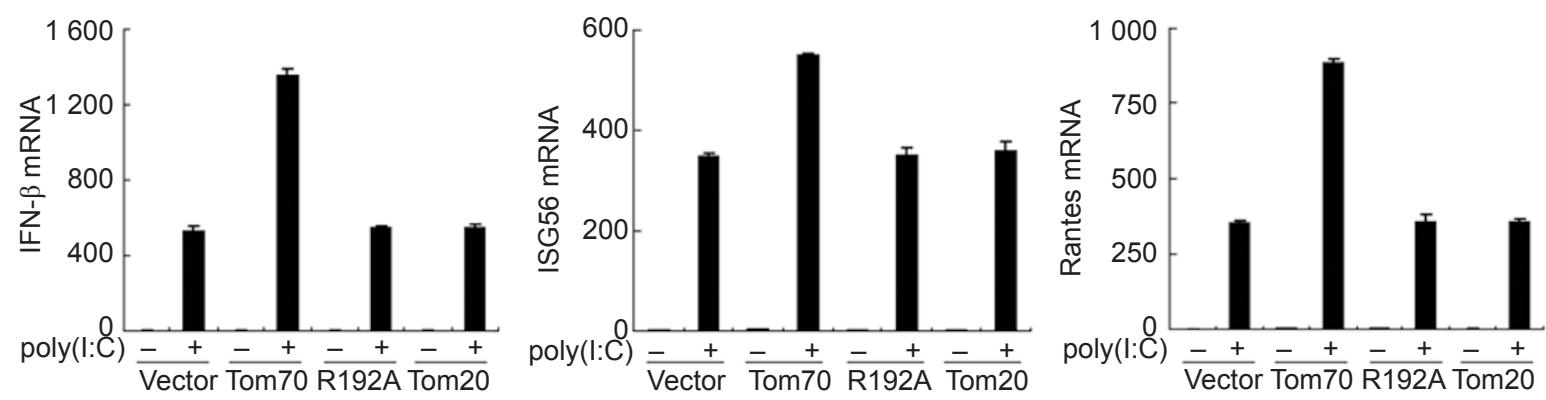

C

BMDM
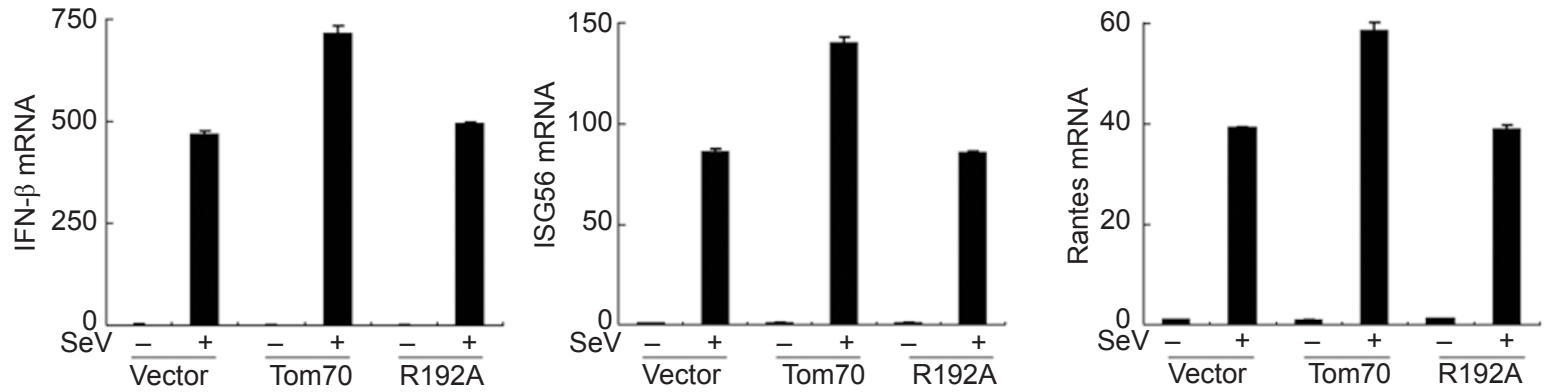

D

BMDC
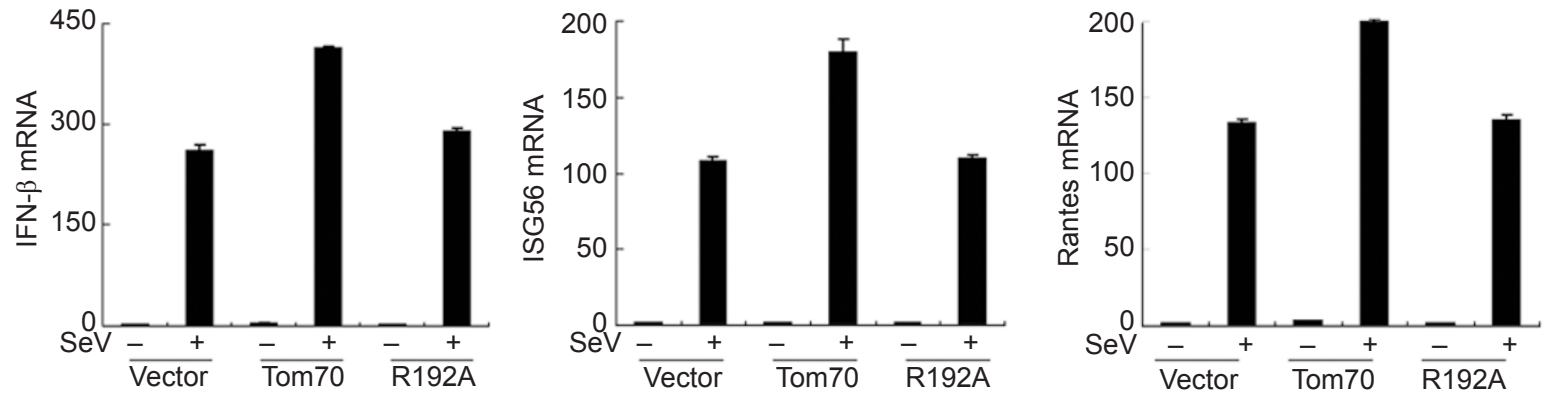

Figure 2 Tom70 synergizes IRF3 activation during SeV infection or poly(I:C) stimulation. (A, B) HEK293 cells were transfected with indicated plasmids and then challenged by SeV infection (A) or poly(I:C) transfection (B). Induction of IFN- $\beta$, ISG56 and Rantes mRNAs was measured by Q-PCR. (C, D) BMDM (C) or BMDC (D) were nucleofected with indicated plasmids and then stimulated by SeV. Induction of IFN- $\beta$, ISG56 and Rantes mRNAs was measured by Q-PCR. Data are presented as means \pm s.d. $(n=3)$. NC, nonspecific control; R192A, the point mutant of Tom70; BMDM, bone marrow-derived macrophage; BMDC, bone marrow-derived dendritic cell. 
(bone marrow-derived macrophages; Figure 2C) and BMDCs (bone marrow-derived dendritic cells; Figure 2D). In addition, Tom70 also potentiated the expression of endogenous NF- $\mathrm{kB}$-responsive genes (Supplementary information, Figure S2). Collectively, these data suggest that Tom70 synergizes the activation of intracellular antiviral signal transduction.

\section{Knockdown of Tom70 impairs the activation of IRF3}

Alternatively, we used the knockdown approach to probe the function of Tom70, using Tom 20 as a negative control. The effective siRNA oligonucleotides were screened out, which could reduce the endogenous protein levels of Tom70 (Supplementary information, Figure S3A) or Tom 20 (Supplementary information, Figure S3B) by $>90 \%$. Initially, luciferase assay was used to evaluate the effect of Tom70 knockdown on IRF3 activation in HEK293 cells. As expected, knockdown of endogenous Tom70 drastically inhibited the expression of both IFN- $\beta$ - and PRDIII-I- luciferase reporters induced by $\mathrm{SeV}$ (Supplementary information, Figure S3C) or transfection of poly(I:C) (Supplementary information, Figure S3D). Similarly, кB-luciferase reporter activity was also impaired by Tom70 knockdown under the same condition (Supplementary information, Figure S3C and S3D). In contrast, both Tom 20 siRNA and Tom $70 \mathrm{mt}$ siRNA, a mutant form of Tom70 siRNA with no silencing activity, failed to do so (Supplementary information, Figure S3C and S3D).

We went on to analyze in HEK293 cells, the effect of Tom70 knockdown on the induction of endogenous IRF3-responsive genes, stimulated by $\mathrm{SeV}$ or poly(I:C). Again, Tom 70 knockdown dramatically inhibited the transcription of IRF3-responsive mRNAs, whereas neither Tom 20 nor Tom70mt siRNA had any inhibitory effect (Figure 3A and 3B). In addition, silencing of Tom70 displayed no effect on TRIF-mediated activation of IFN- $\beta$-luciferase reporters (Supplementary information, Figure S3E) or Wnt signaling (Supplementary information, Figure $\mathrm{S} 3 \mathrm{~F}$ ). We also isolated $\mathrm{P}-\mathrm{M} \varphi$ (peritoneal macrophage), BMDM and $\mathrm{BMDC}$, and respectively transfected these cells with indicated siRNAs followed by $\mathrm{SeV}$ infection. Consistently, knockdown of Tom 70 but not Tom 20 markedly attenuated $\mathrm{SeV}$-induced expression of endogenous IRF3-responsive genes in BMDM (Figure 3C), BMDC (Figure 3D) as well as $\mathrm{P}-\mathrm{M} \varphi$ (Figure 3E). In addition, reduction of endogenous Tom70 displayed the same effects on NF- $\kappa \mathrm{B}$ activation (Supplementary information, Figure S4).

To rule out potential off-target effects of the Tom70 siRNA, an RNAi-resistant Tom70 construct (rTom70) was generated, in which silent mutations were introduced into the sequence targeted by the siRNA without changing the amino acid sequence of the protein. HEK293 cells were first transfected with control or Tom70 siRNA followed by the transfection of control or rTom 70 plasmids as indicated, respectively. Then the induction of IFN- $\beta$ mRNA was measured by Q-PCR after $\mathrm{SeV}$ treatment. As shown in Figure 5F, rescue experiments indicated that $\mathrm{SeV}$-stimulated IFN- $\beta$ mRNA induction was restored by introducing rTom70 into Tom70 knockdown cells. Collectively, these results strongly suggest that Tom70 is a positive regulator of IRF3 activation.

\section{Tom70 functions downstream of MAVS and upstream of TBK 1}

To delineate the topology of Tom70 in RIG-I/MAVS/ TBK1/IRF3 signaling pathway, we noticed that exogenous expression of RIG-I N-terminal tandem CARD domains (RIG-I-N) or MAVS could induce the expression of IFN- $\beta$-luciferase reporters, and this induction was markedly potentiated by the ectopic expression of Tom70 (Figure 4A). Consistently, knockdown of Tom70 impaired the expression of IFN- $\beta$ reporter under the same condition (Figure 4B). However, neither ectopic expression nor knockdown of Tom70 had any effect on the induction of IFN- $\beta$ reporter, when stimulating with exogenous TBK1 (Figure 4A and 4B).

To substantiate the above observations, we investigated the kinase activity of TBK1 and phosphorylation status of IRF3 during $\mathrm{SeV}$ infection, when endogenous Tom70 was knocked down. As shown in Figure 4C, decrease in endogenous Tom70 expression caused significant loss of the kinase activities of TBK1, whereas this effect did not apply to Tom 20 knockdown. Furthermore, reduction of endogenous Tom70 expression also resulted in the significant attenuation of IRF3 phosphorylation induced by SeV (Figure 4D). Taken together, these results suggest that Tom70 functions downstream of MAVS and upstream of TBK1.

As Tom70 is involved in mitochondrial protein transport, it was possible that Tom70 affected the sub-cellular location of MAVS or the integrity of mitochondria. We utilized both sub-cellular fractionation methods and confocal imaging techniques to check where MAVS localized in the absence of Tom70. The steps to isolate the crude mitochondria pellets were shown in the diagram (Figure 4E left panel). It was observed that silencing of endogenous Tom70 did not influence the mitochondrial targeting of MAVS, either endogenously (Figure 4E middle panel) or exogenously (Figure 4E right panel). Confocal imaging also confirmed this observation (Figure $4 \mathrm{~F})$. Furthermore, knockdown of Tom70 did not affect UV-induced apoptosis (Figure 4G). Tetramethylrhod- 
A

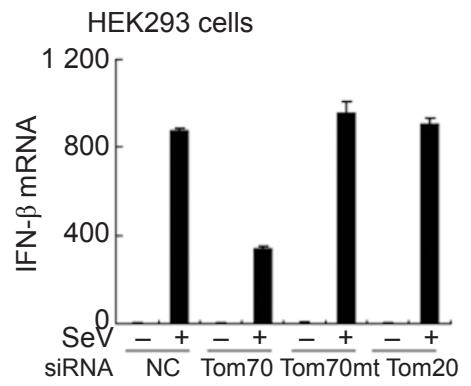

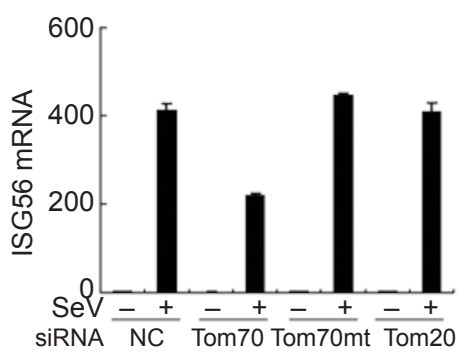

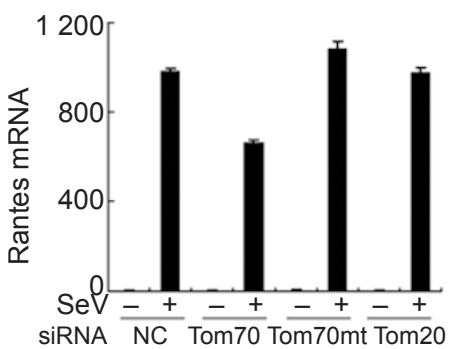

B

HEK293 cells
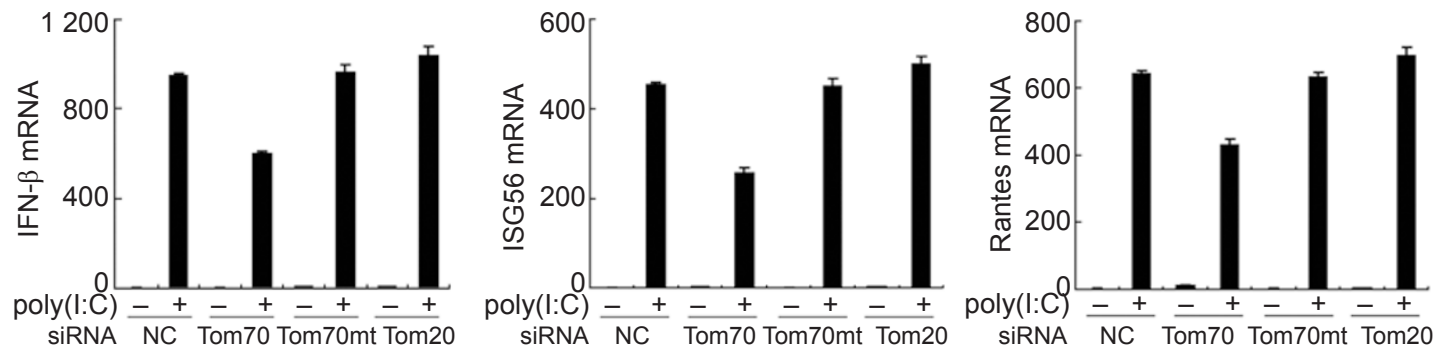

C

BMDM
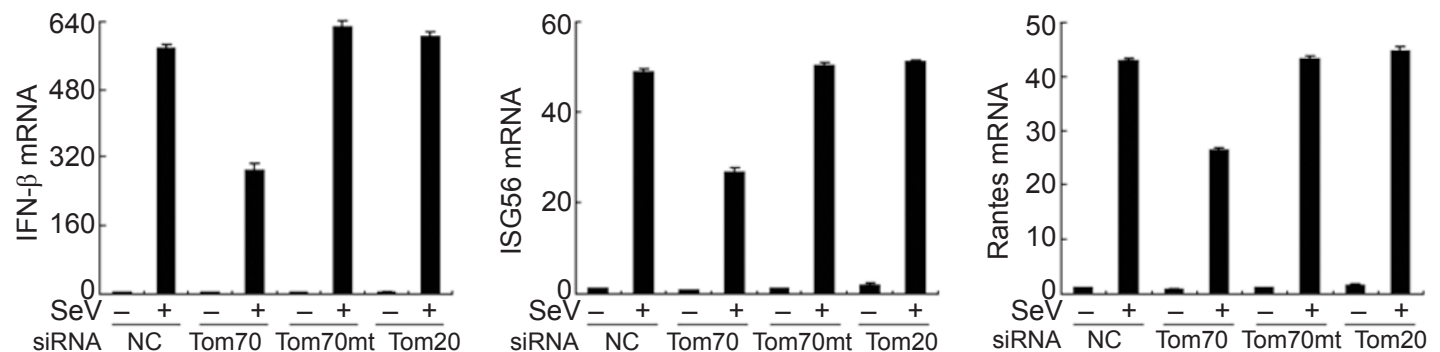

D

BMDC
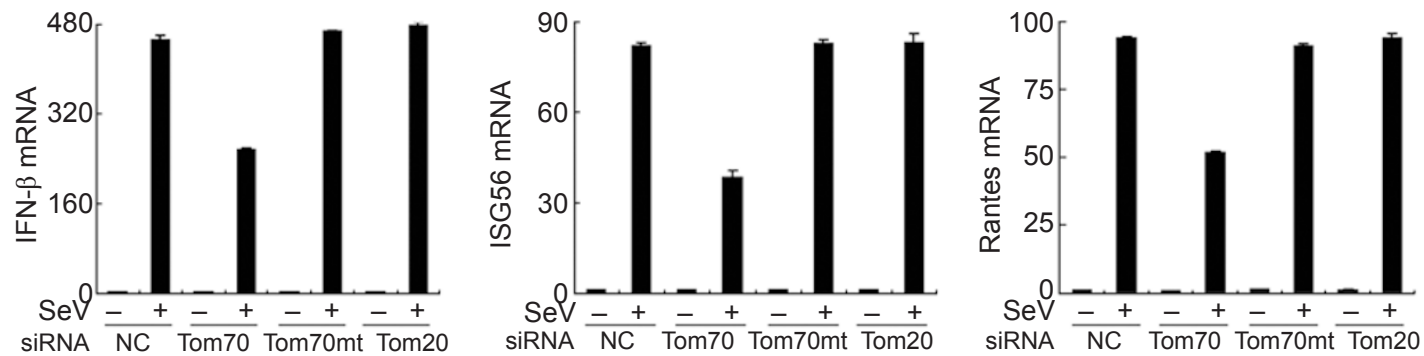

E
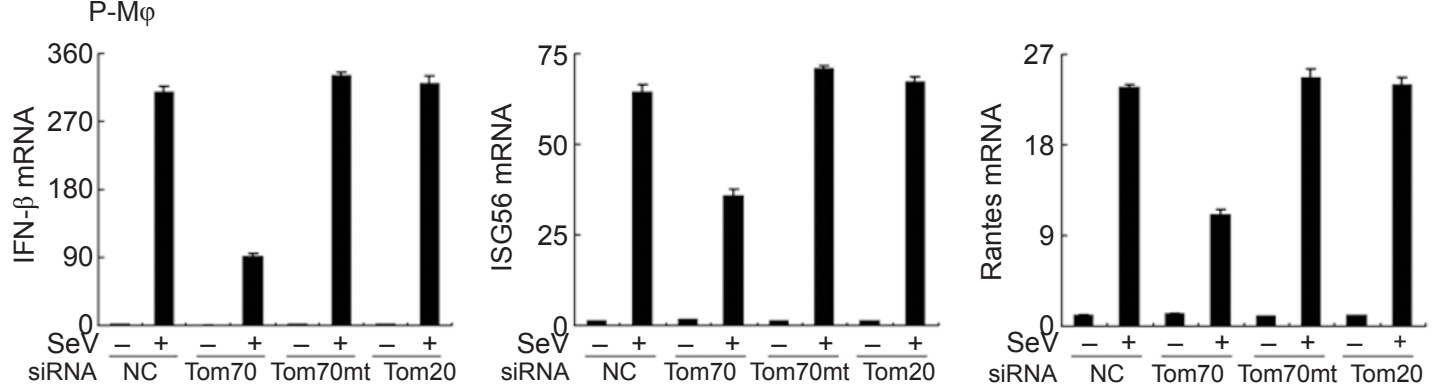
amine methylester (TMRM) staining indicated that the mitochondrial membrane potential remained intact when silencing endogenous Tom70. In contrast, the mitochondrial inhibitor carbonyl cyanide $m$-chlorophenyl hydrazone (CCCP) dramatically blocked TMRM accumulation in mitochondria by disturbing mitochondrial membrane potential (Figure 4H). Collectively, Tom70 plays a specific role during the primary induction of type-I interferons.

Tom70 interacts with MAVS during virus infection or $\operatorname{poly}(I: C)$ stimulation

To confirm the specific interactions between MAVS and Tom70, HA-Tom70 and Flag-MAVS were cotransfected into HEK293T cells, which were then treated by $\mathrm{SeV}$ infection or poly(I:C) transfection for 0-5 h. Cell lysates were immunoprecipitated with either mouse control IgG or anti-Flag antibody. As expected, HA-Tom70 could be co-immunoprecipitated with Flag-MAVS, but not with control $\operatorname{IgG}$ (Figure 5A). Apparently, $\mathrm{SeV}$ or poly(I:C) challenge could enhance the affinity between Flag-MAVS and HA-Tom70 (Figure 5A). Consistently, endogenous Tom 70 co-immunoprecipitated with endogenous MAVS, and this interaction was also enhanced upon $\mathrm{SeV}$ stimulation or poly(I:C) transfection (Figure 5B).

To explore the functional significance of this interaction between Tom70 and MAVS, we generated two mislocation mutants of Tom 70 . Tom $70(\Delta \mathrm{TM})$ was constructed by deleting the $\mathrm{N}$-terminal transmembrane domain, and Tom70(ER-TM) was constructed by replacing its transmembrane domain with a targeting sequence to endoplasmic reticulum (ER; Figure 5C upper panel). Tom70( $\triangle \mathrm{TM})$ and Tom70(ER-TM) were confirmed to localize in the cytosol or ER, respectively (Figure 5C middle and lower panel). Interestingly, Tom70 $(\Delta \mathrm{TM})$ (Figure 5D) and Tom70(ER-TM) (data not shown) failed to interact with wild-type MAVS. Conversely, $\operatorname{MAVS}(\Delta \mathrm{TM})$, a previously reported mutant that localizes in the cytosol, also could not interact with wild-type Tom70 (Figure 5D). These data indicate that the transmembrane domains of both MAVS and Tom70 are essential to mediate their interactions during viral infection.

To corroborate the activity of the mislocation mutants, neither Tom70( $\Delta \mathrm{TM})$ nor Tom70(ER-TM) could potenti- ate the expression of IFN- $\beta$ induced by $\mathrm{SeV}$ or poly(I:C) (Figure 5E). Furthermore, the corresponding RNAi-resistant constructs of Tom70( $\triangle \mathrm{TM})$ and Tom70(ER-TM) were generated, namely rTom70 $(\Delta \mathrm{TM})$ and rTom70(ERTM). Neither of these proteins could rescue the expression of IFN- $\beta$ induced by $\mathrm{SeV}$, after the endogenous Tom70 was knocked down (Figure 5F). Collectively, these data indicated that Tom70 interacts specifically with MAVS during RNA viral infection. It is necessary for both the proteins to localize on the mitochondria for mediating the antiviral responses.

\section{Tom70 recruits TBK1/IRF3 to mitochondria by binding} to Hsp90

Recently, we reported that chaperone Hsp90, IRF3 and TBK1 were constitutively in a protein complex [28]. In addition, a recent study suggested that Hsp90 could deliver a subgroup of mitochondrial pre-proteins, synthesized in the cytosol, to Tom70 for further sorting into mitochondrial compartments [25]. In light of our above results, we speculated that Tom 70 could dynamically recruit TBK1/IRF3 to mitochondria by interacting with Hsp90. To address this hypothesis, ectopically expressed Hsp90 was confirmed to bind Tom70 (Figure 6A lower left panel), and Hsp90 also interacted with IRF3 and TBK1 (Figure 6A lower left panel).

It was suggested that the clamp and core domain of Tom70 (Figure 5C) were responsible for the binding to Hsp90 and mitochondrial pre-proteins, respectively. We mapped the clamp domain of Tom70 and C-terminal motif of Hsp90 to mediate this interaction (data not shown). In addition, Hsp90 consists serially of three domains (Figure 6A upper panel). The N-terminal domain contains an ATP-binding site, while the middle domain displays high affinity for its client proteins. Consequently, several mutants of Tom70 and Hsp90 were generated as described, so that Tom70(R192A) had a point mutation in the clamp domain (Figure 5C), while the EEVD motif of Hsp90 was mutated to EEAA for Hsp90(2A) or truncated for $\operatorname{Hsp} 90(\Delta 4)$ (Figure 6A upper panel). We confirmed that the sub-cellular locations of Tom70(R192A) remained the same as the wild-type (Figure 1D). Therefore, wild-type or mutant forms of HA-Hsp90 and FlagTom70 were ectopically expressed in different combina-

Figure 3 Knockdown of Tom70 impairs IRF3 activition upon challenging by SeV or poly(I:C). (A, B) HEK293 cells were transfected with indicated siRNA and challenged by SeV infection (A) or poly(I:C) transfection (B). Then induction of IFN- $\beta$, ISG56 and Rantes mRNAs was measured by Q-PCR. (C-E) BMDM (C), BMDC (D) or P-M $\varphi$ (E) were transfected with indicated siRNA and then stimulated by SeV. Induction of IFN- $\beta$, ISG56 and Rantes mRNAs was measured by Q-PCR. Data are presented as means \pm s.d. $(n=3)$. NC, nonspecific control; BMDM, bone marrow-derived macrophage; BMDC, bone marrowderived dendritic cell; $\mathrm{P}-\mathrm{M} \varphi$, peritoneal macrophage. 
A

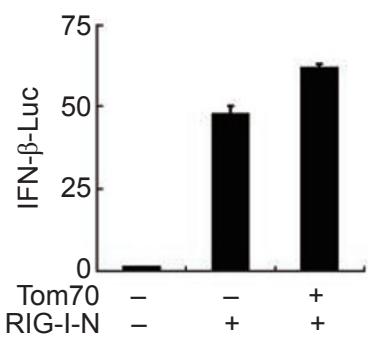

B

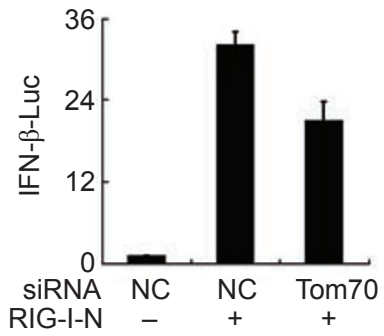

C

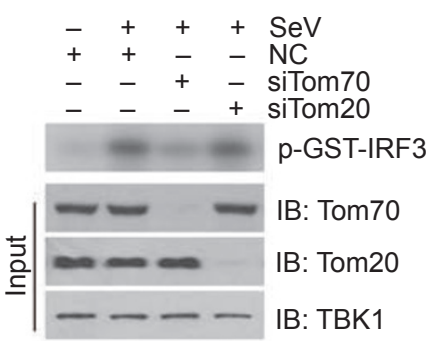

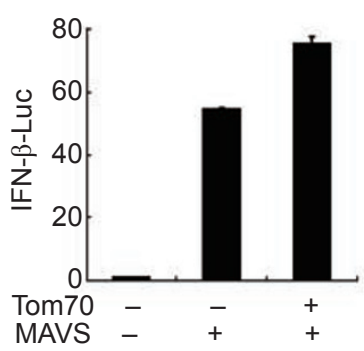
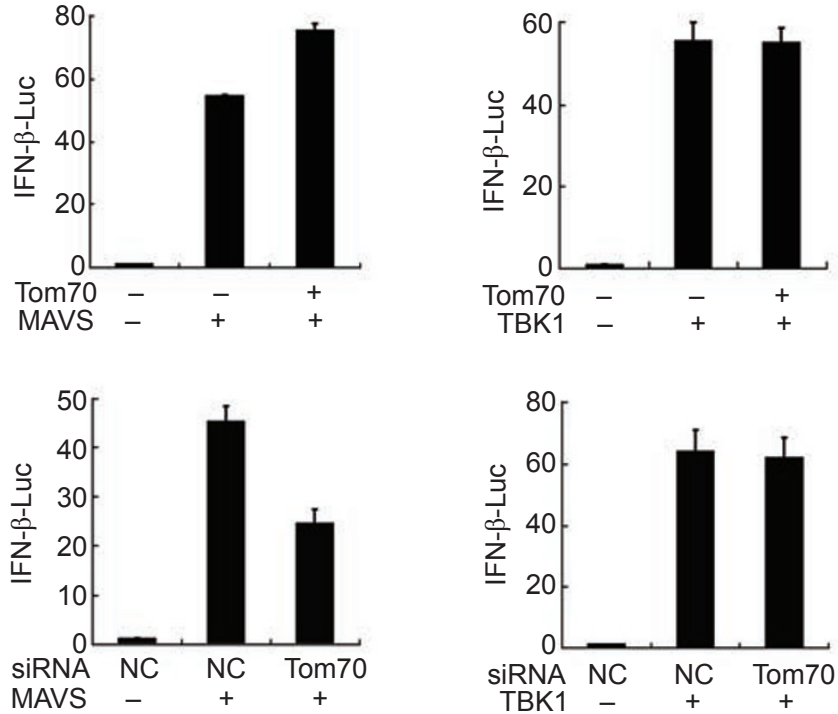

E

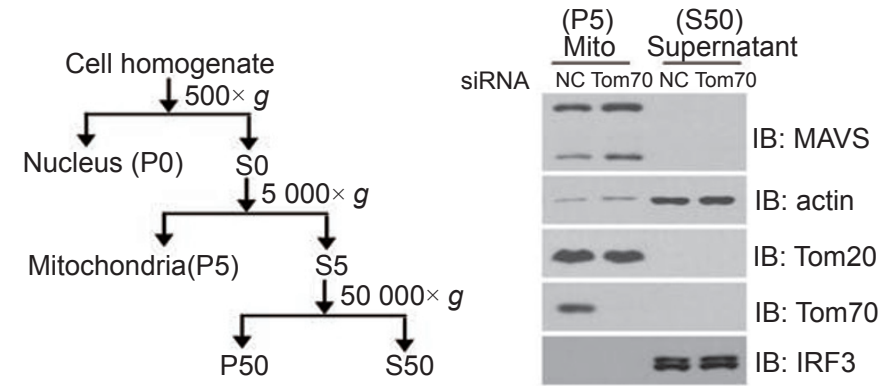

$\begin{array}{lllllllll}0 & 1 & 2 & 4 & 0 & 1 & 2 & 4 & \mathrm{SeV}\end{array}$

$\begin{array}{lllllllll}+ & + & + & + & - & - & - & - & \text { NC } \\ - & - & - & - & + & + & + & + & \text { siTom70 }\end{array}$
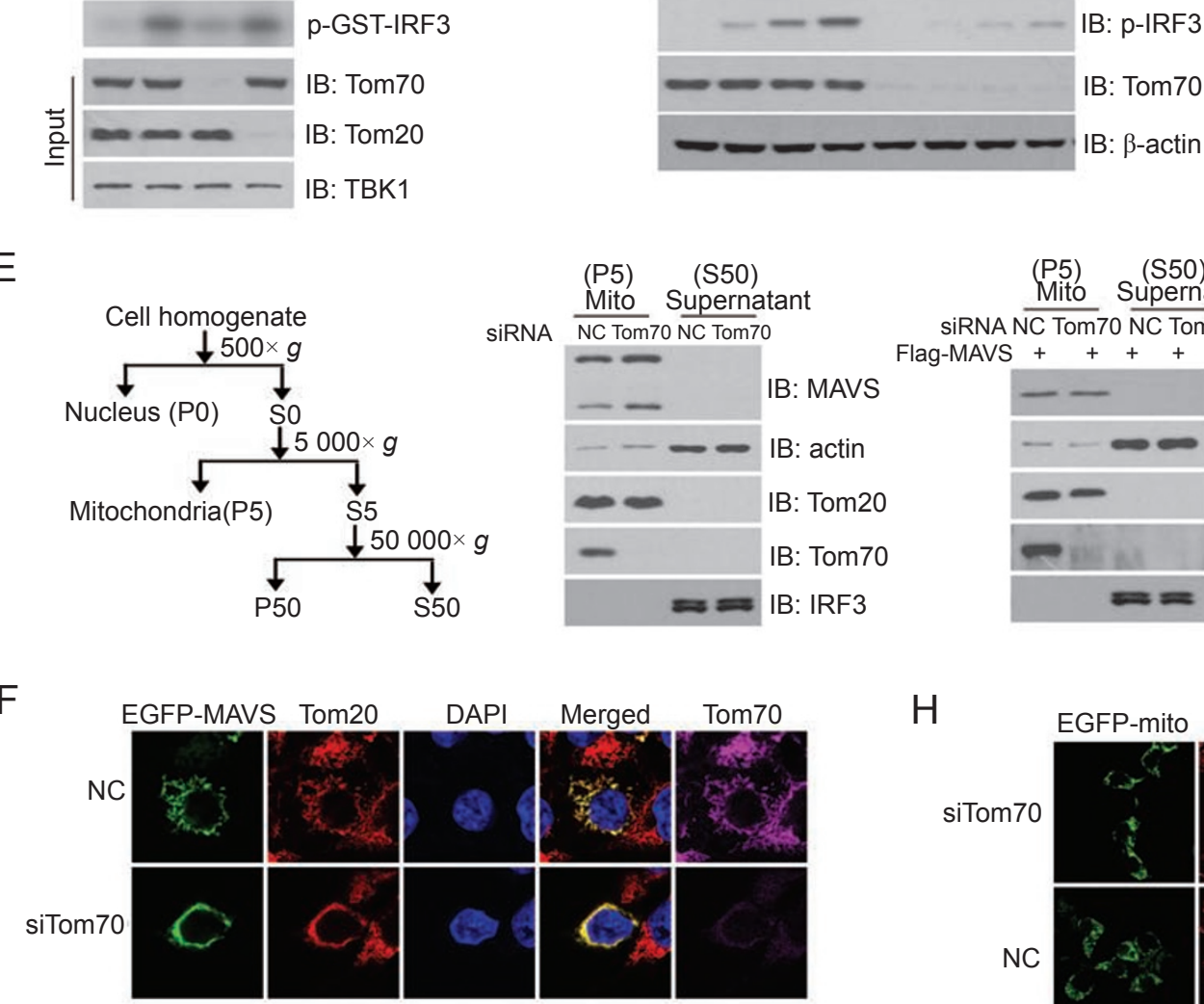

$\mathrm{H}$ siRNA NC Tom70 NC Tom70 Flag-MAVS

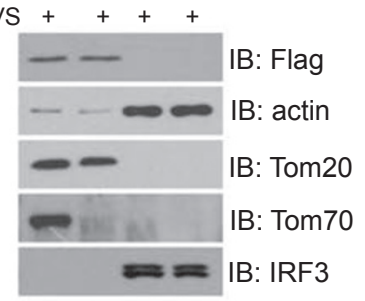

G

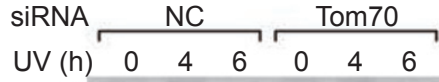

Full-length $\rightarrow$

IB: PARP

Processed -

IB: actin

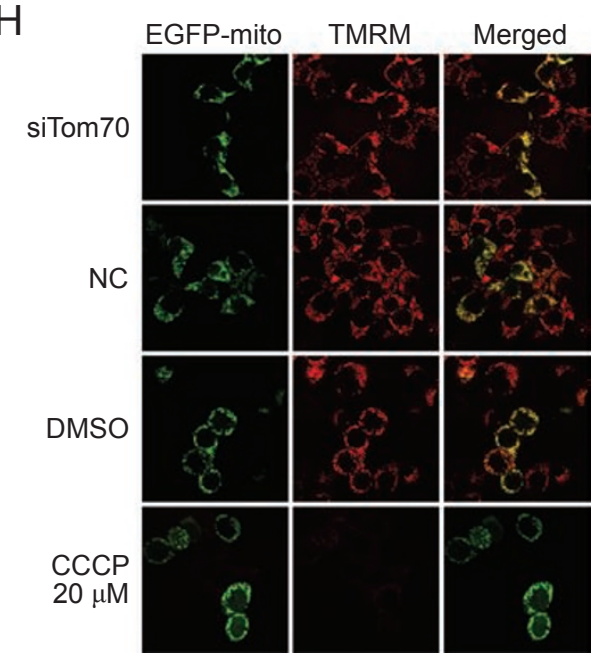


tions and subjected to co-immunoprecipitation assay. It was revealed that Flag-Tom70(R192A) did not interact with HA-Hsp90; conversely, neither HA-Hsp90(2A) nor HA-Hsp90 $(\Delta 4)$ could interact with Flag-Tom70 (Figure $6 \mathrm{~A}$ lower right panel).

Endogenously, Tom70 interacted strongly with Hsp90; Tom70 also co-immunoprecipitated with IRF3 and TBK1 (Figure 6B). In contrast, Tom 20 did not bind to Hsp90, IRF3 or TBK1 (Figure 6B). To explore whether Hsp90 served as a bridge, we used RNAi strategy to effectively reduce the amount of endogenous Hsp90 (Supplementary information, Figure S5). It was found that the ability of Tom70 to co-immunoprecipitate IRF3 or TBK1 was apparently reduced when knocking down endogenous Hsp90 (Figure 6C). Conversely, IRF3 could barely coimmunoprecipitate with Tom70 upon decrease of endogenous Hsp90 expression (Figure 6D).

To recapitulate the observations in vitro, GST pulldown assay was performed using wild-type or mutant forms of recombinant Hsp90, Tom70 and IRF3 proteins. First, we pulled down His-IRF3 with wild-type or mutant forms of GST-Hsp90. As shown in Figure 6E upper left panel, Hsp90 interacted directly with His-IRF3. Perturbation of the Hsp90 C-terminal motif (GST-Hsp90(2A) and GST-Hsp90( $\Delta 4)$ ) did not affect its binding to IRF3. Then, we confirmed the direct interaction between HisHsp90 and GST-Tom70( $\triangle \mathrm{TM})$. Notably, point mutants of either Tom70(R192A) or Hsp90(2A) failed to interact directly with the reciprocal wild-type proteins, which was consistent with the above in vivo results (Figure 6E upper right panel). Finally, we tested whether Hsp90 was critical to mediate the association between Tom70 and IRF3 in vitro. As expected, in the presence of Hsp90, wild-type GST-Tom70( $\Delta \mathrm{TM})$ could pull down His-IRF3. However, when crippling the direct interaction between GST-Tom 70( $\Delta \mathrm{TM})$ and His-Hsp90 by using the corresponding mutants, it was found that Tom70 completely failed to pull down IRF3 (Figure 6E lower panel). Taken together, these data indicated that Tom 70 recruits TBK1/ IRF3 via Hsp90, both in vivo and in vitro.

The direct interaction between Tom70 and Hsp90 is essential for IRF3 activation

To further explore the physiological relevance of this direct interaction, we observed that Tom70(R192A), which was deprived of the ability to bind Hsp90, could not potentiate the expression of IRF3-responsive reporters stimulated by $\mathrm{SeV}$ infection or poly(I:C) (Supplementary information, Figure S1C and S1D), although Tom70(R192A) was expressed similarly to wild-type Tom70 on mitochondria (Figure 1D). Similarly, the induction of IRF3-mediated endogenous genes by $\mathrm{SeV}$ or poly(I:C) was not enhanced by ectopically expressing this Tom70 mutant in either HEK293 or primary cells (BMDM or BMDC; Figure 2A-D). Moreover, rTom70(R192A), the corresponding RNAi-resistant construct, failed to rescue IFN- $\beta$ expression induced by $\mathrm{SeV}$, after attenuating endogenous Tom70 expression (Figure $5 \mathrm{~F})$.

We confirmed that decrease in endogenous Hsp90 expression by siRNA resulted in significant reduction of the IFN- $\beta$ gene transcription induced by $\mathrm{SeV}$ infection or poly(I:C) transfection (Figure $6 \mathrm{~F}$ ). We also ruled out the potential off-target effects of the Hsp90 siRNA by demonstrating that rHsp90, the RNAi-resistant wildtype Hsp90, could restore $\mathrm{SeV}$-stimulated IFN- $\beta$ mRNA

Figure 4 Tom70 functions downstream of MAVS and upstream of TBK1. (A) IFN- $\beta$-luc and pTK-Renilla reporters were transfected into HEK293T cells together with empty vector or Tom70 plasmids, and then challenged by RIG-I-N, MAVS or TBK1 constructs. (B) HEK293T cells were transfected and stimulated as in (A), except that siRNA were transfected in lieu of plasmids. RIG-I-N, the N-terminal of RIG-I (1-286 aa) containing the tandem CARD domains. (C) HEK293 cells were transfected with indicated siRNA and then stimulated with or without SeV for $2 \mathrm{~h}$. Equal amount of cell lysates were immunoprecipitated with anti-TBK1 antibody and subjected to kinase assay using GST-IRF3 and $\gamma-{ }^{32}$ P-ATP as substrates. (D) HEK293 cells were transfected with control or Tom70 siRNA and then stimulated with SeV for indicated time. Cell lysates were resolved in SDSPAGE followed by immunoblotting with indicated antibodies. (E) HEK293T cells were treated with control or Tom70 siRNA for $48 \mathrm{~h}$ and fractionated as in diagram (left panel). The corresponding fractions were analyzed by immunoblotting with indicated antibodies (middle panel). On the right panel, HEK293T cells were transfected with Flag-MAVS, along with control or Tom70 siRNA. The cells were fractionated and immunoblotted as indicated. (F) HEK293T cells were transfected with EGFP-MAVS, along with control or Tom70 siRNA. The cells were stained with indicated antibodies and imaged by confocal microscopy. (G) HEK293 cells were transfected with indicated siRNA for $48 \mathrm{~h}$. Then the cells were irradiated with UV $\left(80 \mathrm{mj} / \mathrm{cm}^{2}\right)$ and incubated for the indicated time periods. Cell lysates were immunoblotted with indicated antibodies. (H) Indicated siRNAs were transfected into HEK293T cells together with EGFP-mito plasmid (upper two panels); the cells were then incubated with 10 $\mu \mathrm{M}$ TMRM for $30 \mathrm{~min}$ to monitor the mitochondrial membrane potential. As controls, HEK293T cells transfected with EGFPmito plasmid were treated with DMSO or mitochondrial inhibitor CCCP (lower two panels) and then subjected to TMRM staining as above. NC, nonspecific control; CCCP, carbonyl cyanide m-chlorophenyl hydrazone; TMRM, tetramethylrhodamine methylester. 
A

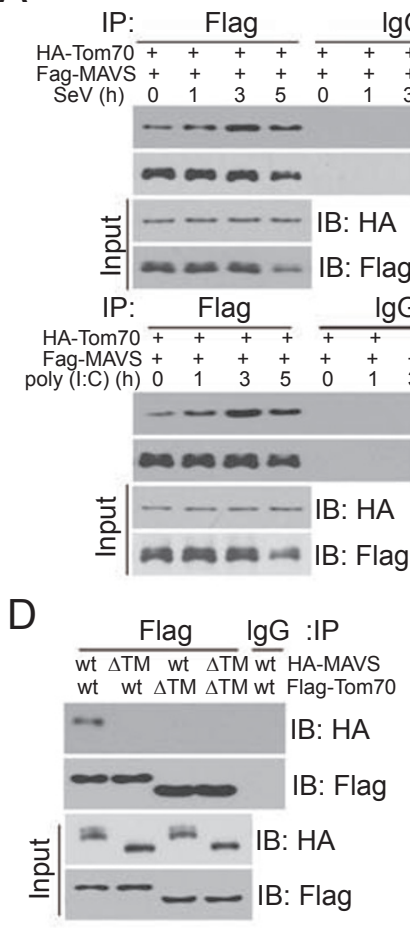

B

IB: HA

IB: Flag

- - - IB: MAVS

IP: $\frac{\text { Tom70 }}{0} \frac{\operatorname{lgG}}{0}$

IB: HA

IB: Flag

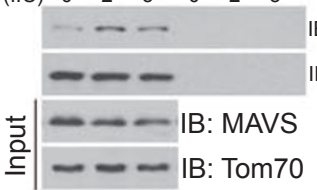

E
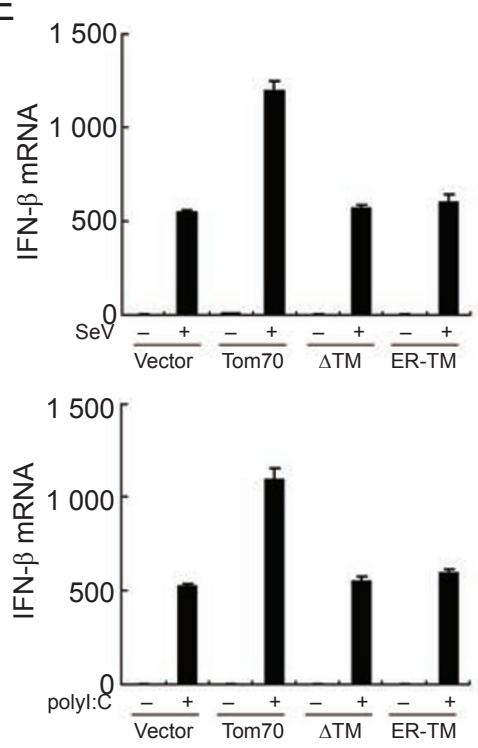

C

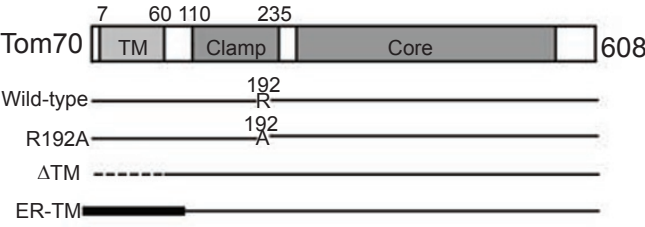

Anti-HA Anti-calnexin DAPI Merged B: MAVS Tom70-HA B: Tom70 (ER-TM)
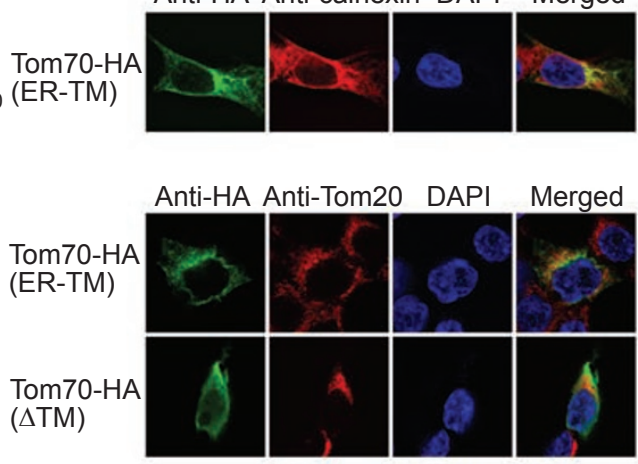

F

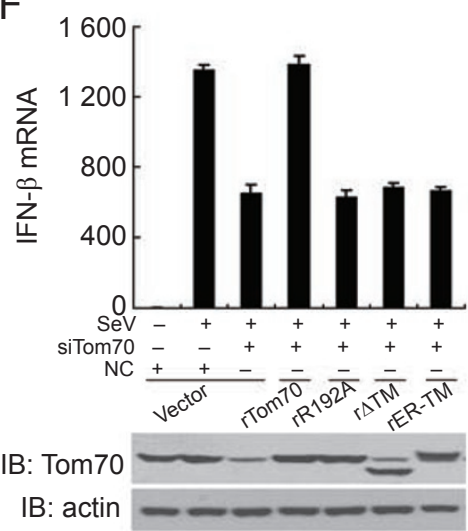

Figure 5 Tom70 interacts specifically with MAVS on mitochondria during viral infection. (A) HEK293T cells were co-transfected with MAVS and Tom70, and challenged by SeV or poly(l:C) for indicated time and then subjected to immunoprecipitation with control IgG or anti-Flag antibody. (B) HEK293 cells were challenged by SeV or poly(I:C) for indicated time and then subjected to immunoprecipitation with control IgG or anti-Tom70 antibody. (C) Tom70 mutants ( $\triangle T$ M or ER-TM, upper panel) were transfected individually into HEK293T cells, stained by anti-HA along with anti-calnexin (middle panel) or anti-Tom20 (lower panel) antibodies and then imaged by confocal microscopy. (D) HEK293T cells were co-transfected with indicated constructs and then subjected to immunoprecipitation with control IgG or anti-Flag antibody. (E) Induction of IFN- $\beta$ mRNA by SeV infection or poly(I:C) transfection in the presence of indicated plasmids were measured by Q-PCR. (F) HEK293 cells were transfected with control or Tom70 siRNA, and then rescued with indicated siRNA-resistant Tom70 constructs. IFN- $\beta$ mRNA induced by SeV was measured by Q-PCR. Data are presented as means \pm s.d. $(n=3)$. NC, nonspecific control; TM, transmembrane domain; ER-TM, Tom70 having its transmembrane domain swapped with an endoplasmic reticulum targeting sequence.

expression in Hsp90 knockdown cells (Figure 6G). Then, we generated RNAi-resistant Hsp90 mutants in the background of $\mathrm{Hsp} 90(2 \mathrm{~A})$ and $\mathrm{Hsp} 90(\Delta 4)$, namely rHsp90(2A) and $\mathrm{rHsp} 90(\Delta 4)$, so that $\mathrm{rHsp} 90(2 \mathrm{~A})$ and rHsp90 $(\Delta 4)$ could remain intact when knocking down endogenous Hsp90, whereas they expressed the same protein as $\mathrm{Hsp} 90(2 \mathrm{~A})$ and $\mathrm{Hsp} 90(\Delta 4)$, respectively. Obviously, neither rHsp90(2A) nor $\mathrm{rHsp} 90(\Delta 4)$ could bind to Tom70. Consistent with this, it was observed that neither $\mathrm{rHsp} 90(2 \mathrm{~A})$ nor $\mathrm{rHsp} 90(\Delta 4)$ could rescue the expression of IFN- $\beta$ mRNA stimulated by $\mathrm{SeV}$, when knocking down endogenous Hsp90 (Figure 6G). 
A
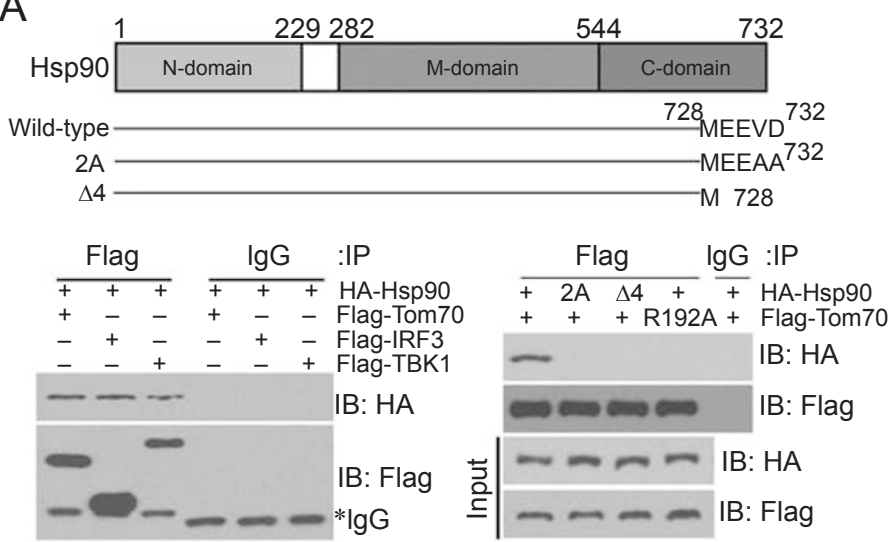

E
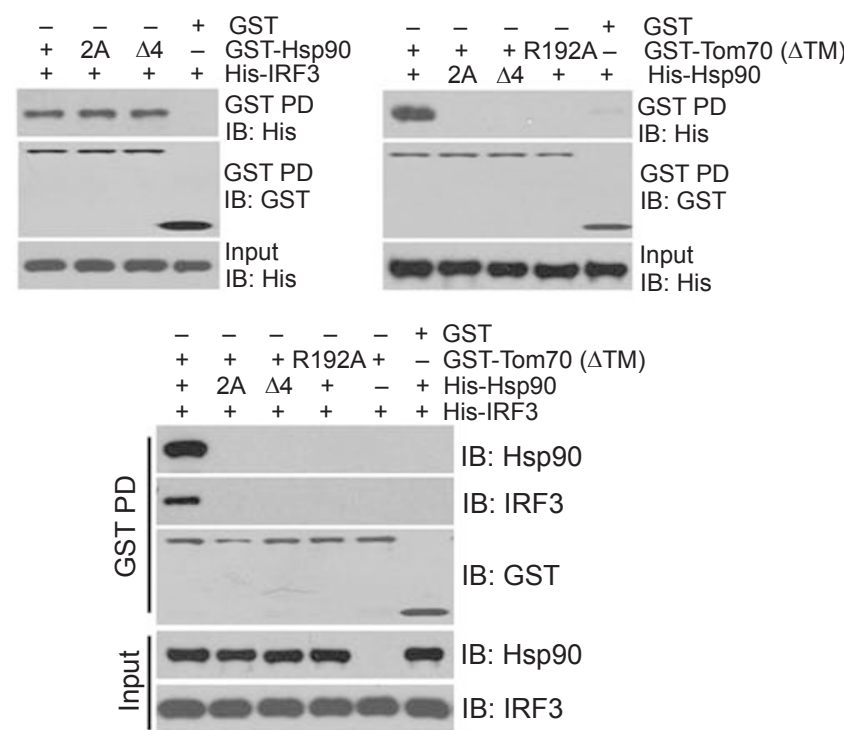

F

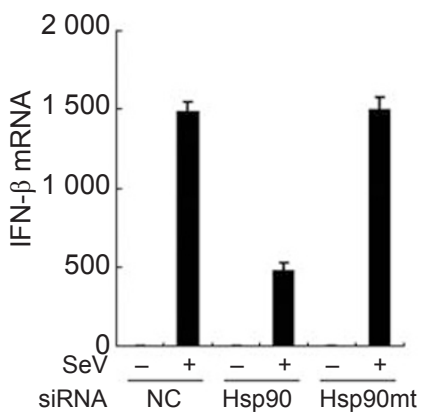

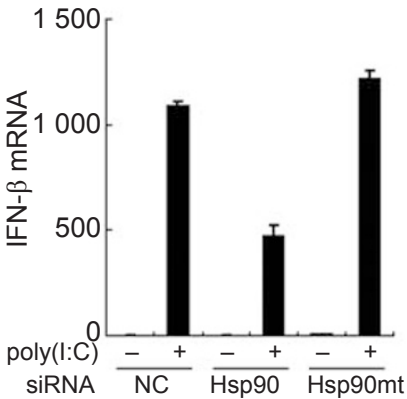

B

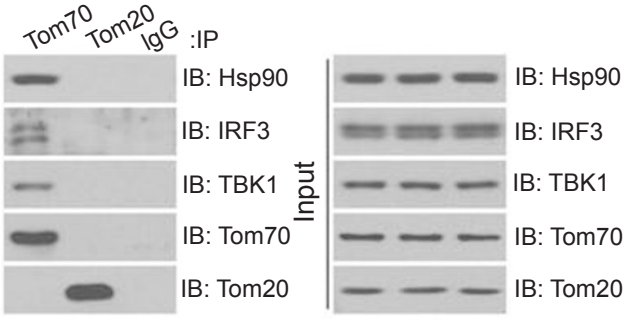

C

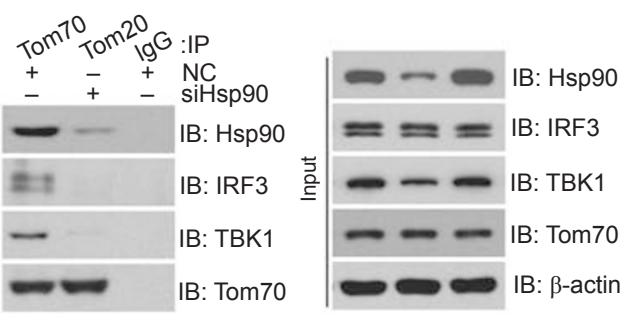

D IRF3 IRF3 IgG :IP

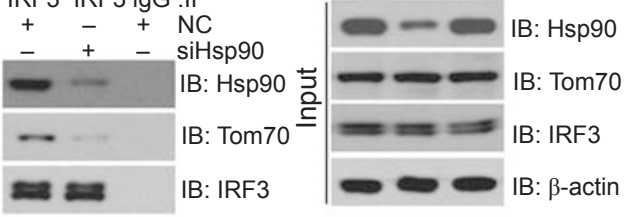

G

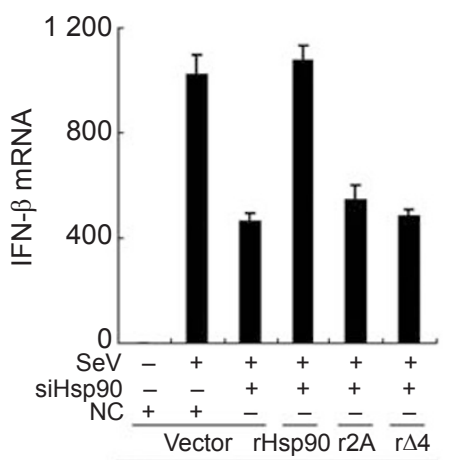

IB: $\mathrm{Hsp} 90=-2= \pm=$

IB: $\beta$-actin ----

Figure 6 Tom70 recruits Hsp90/TBK1/IRF3 complex to mitochondria during viral infection. (A) HEK293T cells were co-transfected with indicated constructs and then subjected to immunoprecipitation with control lgG or anti-Flag antibody. (B) Equal amount of HEK293T cell lysates were subjected to immunoprecipitation with indicated antibodies. (C, D) HEK293T cells were treated with control or Hsp90 siRNA and then subjected to immunoprecipitation with control IgG, anti-Tom70 (C) or anti-IRF3 (D) antibody as indicated. (E) GST pull-down assay was performed as described in methods. Equal amount of recombinant proteins (wild-type or mutants) were added according to indicated combinations to explore the direct interaction between GST-Tom70 and His-Hsp90 (upper left panel), or between GST-Hsp90 and His-IRF3 (upper right panel), or between GST-Tom70, His-Hsp90 and His-IRF3 (lower panel). (F) Induction of IFN- $\beta$ mRNA by SeV infection or poly $(\mathrm{I}: \mathrm{C})$ transfection in the presence of indicated siRNA was measured by Q-PCR. (G) HEK293 cells were transfected with control or Hsp90 siRNA and then rescued with indicated siRNA-resistant Hsp90 constructs. After SeV infection, induction of IFN- $\beta$ mRNA was measured by Q-PCR. Data are presented as means \pm s.d. $(n=3)$. NC, nonspecific control. 
Tom70 enhances IRF3-mediated antiviral responses

Finally, we studied the physiological function of Tom70 during primary host antiviral responses. It is well known that the induction of IFN- $\beta$ is a hallmark of host antiviral abilities. To explore whether Tom70 regulates the production of IFN- $\beta$, we transfected Tom70, Tom70(R192A) or Tom20 into HEK293 cells followed by $\mathrm{SeV}$ treatment. Using enzyme-linked immunosorbent assay (ELISA), we observed that Tom70 could significantly promote IFN- $\beta$ protein production, whereas neither Tom70(R192A) nor Tom20 was able to do so (Figure 7A). Furthermore, knockdown of endogenous Tom70 by introduction of siRNA to HEK293 cells drastically impaired IFN- $\beta$ protein production upon $\mathrm{SeV}$ infection, whereas knockdown of Tom 20 had no apparent inhibitory effect (Figure 7B). As another control, Tom70mt siRNA did not affect IFN- $\beta$ expression either (Figure 7B).

As IFN- $\beta$ could protect cells from infection, we assessed whether Tom 70 played a role in virus restriction. HEK293 cells were transfected with indicated plasmids (Figure 7D) or siRNA (Figure 7E), and then infected with $\mathrm{SeV}$. The culture supernatants from these cells were used to pre-treat fresh HEK293 cells followed by vesicular stomatitis virus (VSV) infection. It was found that knockdown of Tom70 made cells more sensitive to VSV infection (Figure 7E), whereas ectopic expression of Tom70 could protect cells from VSV infection (Figure 7D).

We next investigated whether Tom70 modulated viral replication by challenging the cells directly with VSV or Newcastle disease virus-green fluorescent protein (NDVGFP). HEK293 cells transfected with indicated siRNA were treated with VSV for $16 \mathrm{~h}$, and then the titers of VSV were analyzed by standard plaque assay. As shown in Figure 7C, Tom70 knockdown resulted in a significant increase in virus titer compared to controls. For NDVGFP infection, exogenous expression of Tom70 significantly suppressed NDV-GFP viral replication in HEK293 cells, whereas overexpression of either Tom70(R192A) or Tom 20 had no such function (Figure 7F). Furthermore, depletion of Tom70 markedly augmented the levels of NDV-GFP-positive cells. In contrast, knockdown of Tom20 displayed no apparent increase in NDV-GFP infection (Figure 7G). Conclusively, these data indicate that Tom70 serves as a positive regulator of IRF3 activation and it promotes host antiviral responses during virus infection.

\section{Discussion}

During the past several years, a new paradigm is pro- posed that RIG-I-like helicases and Toll-like receptors (TLRs) detect RNA viruses from topologically intra- and extracellular compartments and initiate immediate host defense to restrict virus infection and proliferation. Simultaneously, cytokines and chemokines are induced to mobilize immune cells to prevent pathological infection and to eliminate invasive virus. An important outcome of RIG-I antiviral signal transduction is the rapid and robust induction of type-I interferons (in particular IFN- $\beta$ ), in which the pivotal process is to activate protein kinase TBK1 and transcription factor IRF3 through the MAVS signal complex on the mitochondrial outer membrane.

Interestingly, MAVS per se localizes on the outer membrane of mitochondria via its C-terminal transmembrane domain (TM) [11]. In contrast, the rest of the reported components in the MAVS signal complex reside in the cytosol. TRAF3 associates with MAVS after dimerization of the MAVS [33]. Consequently, TRAF3 could catalyze the formation of K63 poly-ubiquitin. This process is attenuated by de-ubiquitinase DUBA [34]. In addition, MAVS is recently reported to be modified by both K63 and K48 poly-ubiquitin [21]. Ubiquitination plays both positive and negative roles in regulating the MAVS signal complex $[22,35]$. The relevant molecular mechanisms need further investigation. TRADD and TANK are also reported to be the components of MAVS complex after virus infection. However, a recent study questions the possible role of TANK in RIG-I signaling [36].

Notably, a critical missing link exists as to how MAVS signal complex relays activation signal to TBK1 and IRF3. We hypothesized that there might be unknown adaptor protein(s) on mitochondria to fill this gap. By proteomics approach, we identify Tom70 as the candidate adaptor on mitochondrial outer membrane. Several lines of evidence in this study substantiate the critical function of Tom70 in RIG-I signaling. First, exogenous expression of Tom70 potentiates the induction of IRF3responsive genes upon $\mathrm{SeV}$ infection or poly(I:C) transfection, but does not affect the TRIF-mediated IRF3 activation. Second, knockdown of Tom70 unequivocally results in the significant reduction of IRF3-mediated gene expressions, and this attenuation could be rescued by exogenously expressing a siRNA-resistant rTom70. Third, the kinase activity of TBK1 and the phosphorylation of IRF3 are apparently impaired when knocking down endogenous Tom70 during virus infection, indicating that Tom70 functions upstream of TBK1. Fourth, Tom70 interacts with MAVS. This interaction increases markedly upon virus challenge. Mislocation of Tom70 to either cytosol or ER abolishes its potentiating activity towards IRF3 activation. RNAi-resistant mutants of these 

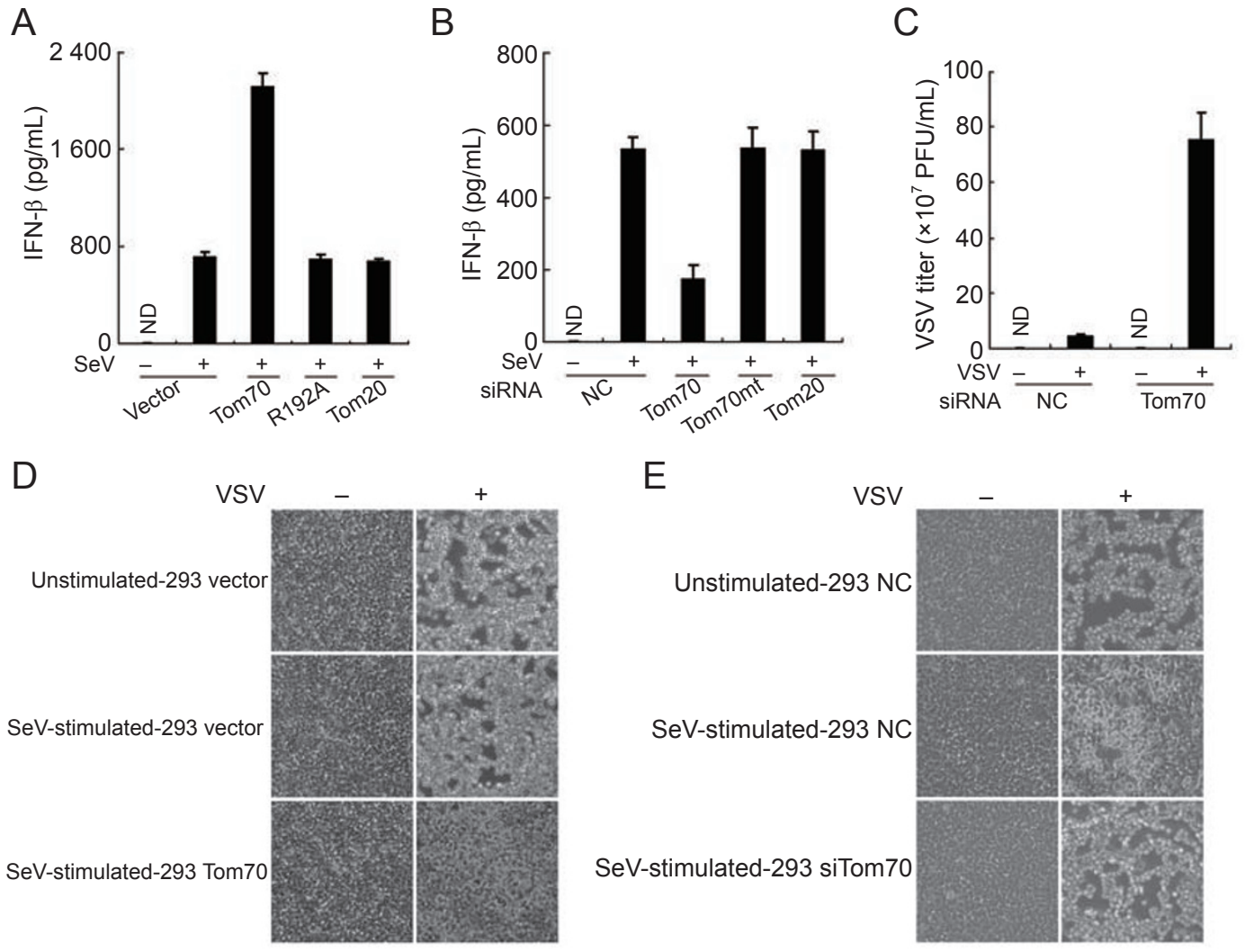

$\mathrm{F}$

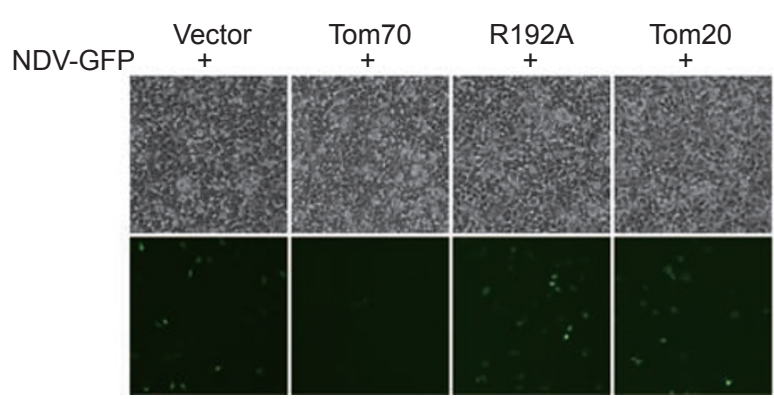

\section{G}

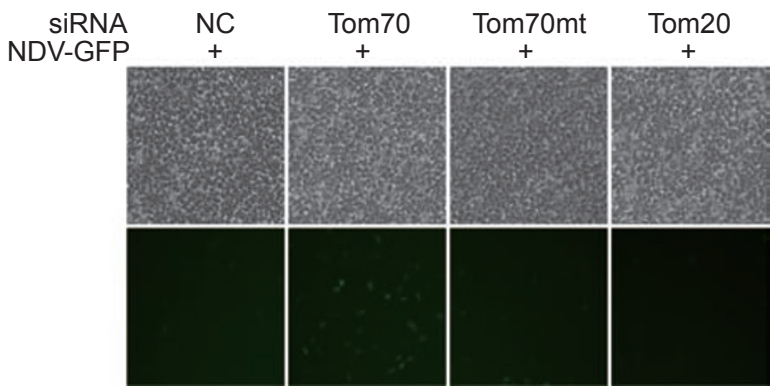

Figure 7 Tom70 modulates IRF3-mediated antiviral responses. (A, B) HEK293 cells were transfected with indicated plasmids (A) or siRNA (B). After SeV infection, IFN- $\beta$ production was determined by ELISA. Data are presented as means \pm s.d. ( $n=3$ ). (C) HEK293 cells transfected with control or Tom70 siRNA were infected with VSV. The titers of VSV were determined by standard plaque assay. Data are presented as means \pm s.d. $(n=3)$. (D, E) HEK293 cells were transfected with indicated plasmids (D) or siRNA (E) and then treated with SeV. Equal volume of culture supernatants from these treatments was applied to fresh HEK293 cells, followed by VSV infection. The cytopathic effect (CPE) was observed by PC microscopy. Original magnification, $\times 40$. (F, G) NDV-GFP replication in HEK293 cells transfected with indicated plasmids (F) or siRNA (G) were visualized by fluorescence microscopy. Data are representative of three independent experiments. NC, nonspecific control; R192A, the point mutant of Tom70.

mislocation constructs also fail to rescue the expression of IFN- $\beta$ in Tom70 knockdown cells. Fifth, gain or loss of Tom70 could respectively promote or impair IFN- $\beta$ production upon $\mathrm{SeV}$ infection, thus boosting or crippling the host antiviral responses.

Previously, we reported that the N-terminal domain of Hsp90 interacted with both TBK1 and IRF3 in resting cell [28]. Disrupting the binding of Hsp90 to TBK1 immediately caused ubiquitin-proteasome-mediated degradation of TBK1. Intriguingly, we observed that the C-terminal truncation mutant of Hsp90(1-620 aa) could bind to TBK1 and IRF3 [28]. However, Hsp90(1-620 
aa) acted as strong dominant-negative mutant for TBK1 activation, suggesting that Hsp90 may link TBK1 and IRF3 to the upstream signaling protein(s). In this study, the clamp domain of Tom70 is uncovered to specifically interact with the C-terminal motif (EEVD) of Hsp90. The importance of this unique interaction is supported by several lines of evidence. Tom70(R192A) does not bind to Hsp90 both in vitro and in vivo, although it is expressed on mitochondria similarly to the wild-type Tom70. Consistently, neither Tom70(R192A) could potentiate IRF3 activation, nor could its RNAi-resistant mutant rTom70(R192A) rescue the expression of IFN- $\beta$ in Tom70 knockdown cells. Conversely, the Hsp90(2A) and Hsp90 $(\Delta 4)$, which are deprived of the ability to bind Tom70, display the same effect as Tom70(R192A). Furthermore, mislocation of Tom70 impairs the activation of TBK1 and IRF3. In conclusion, Tom70 has a novel and essential function in host primary antiviral responses.

Interestingly, Hsp90 was reported to interact strongly with the IKK complex [37]. We confirmed this observation. In addition, we found that Tom70 could immunoprecipitate IKK complex in the presence of Hsp90 (unpublished data; Liu and Wei), indicating that a similar theme applies to the activation of IKK. In light of our current study, a tentative model, on the action of Tom70 is proposed, that Tom 70 recruits both TBK1/IRF3 and IKK complex to mitochondria by specifically recognizing Hsp90, thus linking MAVS to its downstream signaling events (Figure 8). It is a great challenge to elucidate the molecular dynamics of the MAVS/Tom70 signaling complex in future investigations.

Recent studies have revealed two mitochondrial proteins MAVS (also called IPS-1, VISA and Cardif) and NLRX1 to modulate antiviral signal transduction $[9-12,38]$. It is still controversial whether a third protein STING/MITA is localized on mitochondria [30, 31]. An unresolved issue is that why mitochondria is selected as the platform to initiate intracellular antiviral signaling.

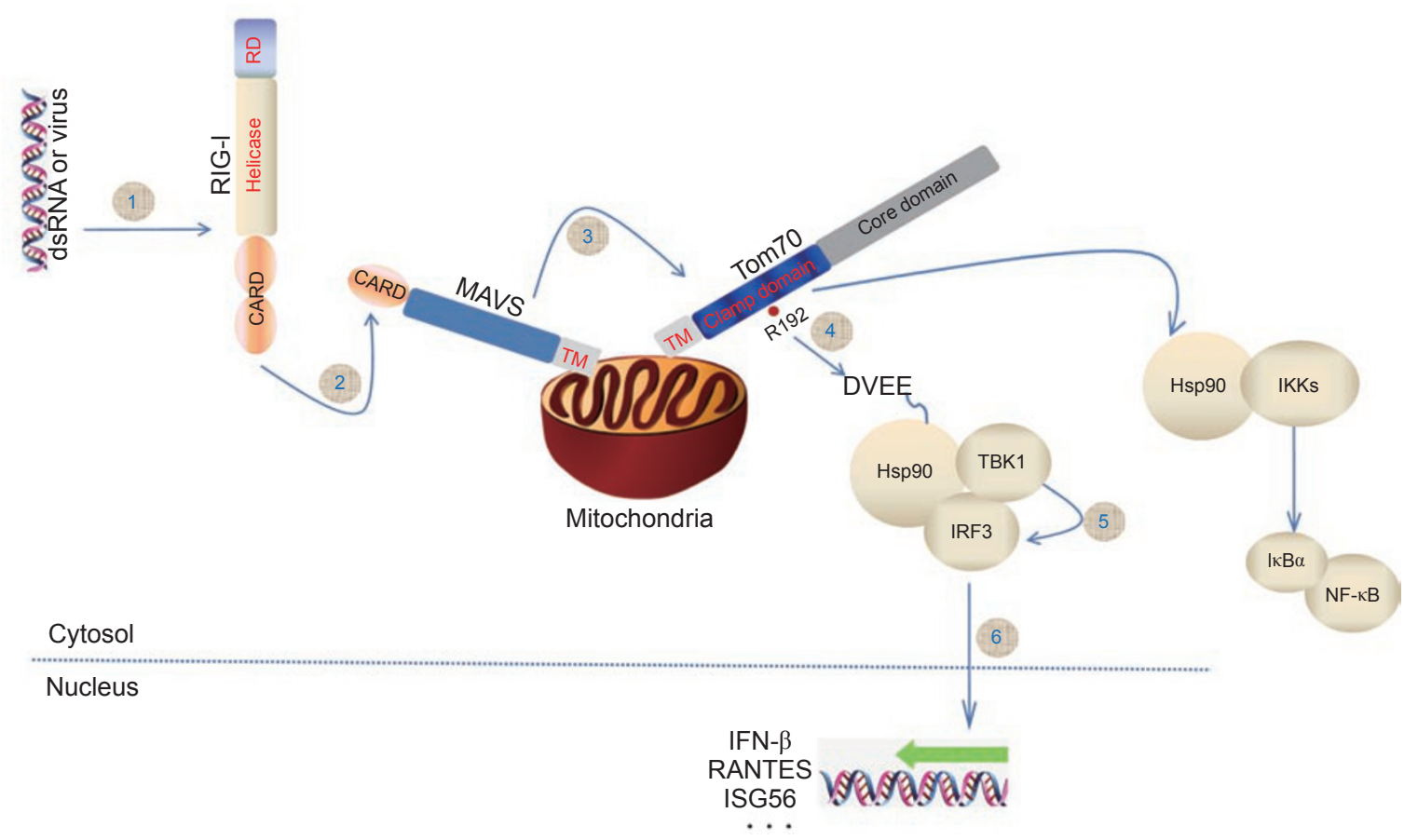

Figure 8 Schematic diagram of the critical role of Tom70 in antiviral signaling. In the cytosol of resting cells, RIG-I is autorepressed in a closed conformation, whereas Hsp90/TBK1/IRF3 form a protein complex constitutively. Upon RNA virus infection, RIG-I binds to double-stranded RNA (dsRNA), which causes RIG-I to undergo dramatic conformational change and results in the exposure of its N-terminal CARD domains (1). Therefore, RIG-I associates with the adaptor protein MAVS on the outer membrane of mitochondria through CARD-CARD homotypic interaction (2). In turn, this triggers MAVS to interact more strongly with Tom70 via their transmembrane domains (3). At the same time, Hsp90 delivers TBK1/IRF3 to mitochondria, via clamp domain of Tom70 and EEVD motif of Hsp90 (4). Transiently, this leads to the formation of a stable signal complex on the mitochondrial outer membrane, including MAVS/Tom70/Hsp90/TBK1/IRF3, inducing a temporary surge of the local concentration of TBK1. Consequently, activated TBK1 phosphorylates the transcription factor IRF3 (5). Ultimately, phosphorylated IRF3 dissociates from the complex and translocates into nucleus to promote antiviral gene transcription (6). Similarly, Hsp90 binds strongly to and delivers IKK complex to Tom70, thus resulting in the activation of IKK and NF- $\mathrm{KB}$. 
The current insight from Tom 70 will probably provide a new clue as to the necessity of forming the signal complex on mitochondria. As Tom70 is conserved from yeast to human, we speculate that the ancient function of Tom70 was dedicated exclusively to recognize a subgroup of mitochondrial proteins synthesized in the cytosol. Probably, evolution took advantage of the unique mechanism that Tom 70 sorts mitochondrial proteins by interacting with Hsp90, in contrast to Tom20 that recognizes a specific signal peptide on the target proteins. In due time, Hsp90 constitutively forms a protein complex with TBK1 and IRF3. Consequently, this protein complex is integrated into innate immunity and recruited to mitochondria for further elaboration of the signal transduction. It will be intriguing to explore when Tom70 gained this new function during the evolution of animal kingdom.

\section{Materials and Methods}

\section{Plasmids}

Human full-length IRF3, TBK1, Hsp90, MAVS and Tom70 cDNAs were cloned from human thymus plasmid cDNA library (Clontech) using standard PCR techniques and then sub-cloned into indicated vectors. All point mutants were generated by using a Quickchange XL (Stratagene). Tom $70(\Delta \mathrm{TM})$ is deprived of its $\mathrm{N}$ terminal transmembrane domain (1-60 aa), while Tom70(ER-TM) replaces its $\mathrm{N}$-terminal transmembrane domain (1-100 aa) with an ER targeting sequence (180 aa). Hsp90( $\Delta 4)$ is truncated of the last four amino acids EEVD at its C-terminus, while Hsp90(2A) has the last two amino acids VD changed to AA at its $\mathrm{C}$-terminus. $\operatorname{MAVS}(\Delta \mathrm{TM})$ is truncated of its C-terminal transmembrane domain (514-540 aa). All Tom70 siRNA-resistant forms were generated by introducing silent mutations in the Tom70 siRNA target sequence (1453-GCG CTG ACG GAC CAG CAA-1470). All Hsp90 siRNA-resistant forms were generated by introducing silent mutations in the Hsp90 siRNA target sequence (1181-GTG ACA AAA AAA AAA AAA AG-1200). All constructs were confirmed by sequencing. pEGFP-mito for labeling mitochondria was kindly provided by Dr Jiansheng Kang (Shanghai Institute for Biological Sciences, SIBS); $\beta$-catenin and TOPflash luciferase reporters were provided by Dr Lin Li (SIBS).

SiRNA

All siRNAs (GenePharma) were transfected using lipofectamine 2000 as described (Invitrogen). HEK293 or 293T cells were plated in 12-well plates in antibiotic-free Dulbecco's modified Eagle's medium (DMEM). At about 40\% confluence, 40-80 pmol siRNA was transfected into cells. To determine efficiency of protein knockdown, at $48 \mathrm{~h}$ post-transfection, cells were lysed in RIPA buffer and immunoblotted with the indicated antibodies.

The siRNA sequences were as follows:

Nonspecific control (NC): 5'-UUC UCC GAA GGU GUC ACG U-3'; Tom70 siRNA: 5'-GGC AUU AAC AGA UCA ACA A-3'; Tom70mt siRNA: 5'-GGC AUU AAA CAG CUA ACA A-3'; Tom20 siRNA human: 5'-CAA GUU ACC UGA CCU UAA A-3'; Tom20 siRNA mouse: 5'-CAA GUU ACC UGA UUU AAA A-3';
Hsp90 siRNA: 5'-ACA AGA AGA AGA AGA AGA A-3'; Hsp90mt siRNA: 5'-ACA AGA AAG GAG AAA AGA A-3'.

\section{Antibodies}

Mouse polyclonal antibody against human Tom70 recombinant protein spanning 110-608 aa was generated in this lab. This antibody was purified by affinity column conjugated with recombinant Tom70 protein, and its specificity was confirmed. Antibodies obtained from commercial sources were as follows: anti-Flag, anti- $\beta$ actin (Sigma); anti-HA, anti-GST, anti-His, anti-IRF3, anti-PARP (Santa Cruz); anti-Hsp90, anti-Tom20 (Proteintech); anti-calnexin, anti-phospho-IRF3(pS386) (Epitomics); anti-TBK1 (Millipore) and anti-MAVS (CST).

\section{Luciferase reporter assays}

Luciferase reporter assays were performed as described previously [28].

\section{Q-PCR}

At 24-48 h after transfection of indicated siRNA or plasmids, HEK293 cells were stimulated with $\mathrm{SeV}$ infection for $8 \mathrm{~h}$ or poly(I:C) transfection for $16 \mathrm{~h}$. Total cellular RNA was isolated with TRIzol (Invitrogen) according to manufacturer's instruction. Reverse transcription of purified RNA was performed using oligodT primer. The quantification of gene transcripts was performed by Q-PCR using SYBR green I dye. All values were normalized to the level of $\beta$-actin mRNA. The primers used were listed in Table 1.

\section{Cell culture}

HEK293T and HEK293 cells were cultured using DMEM plus $10 \%$ FBS, supplemented with $1 \%$ penicillin-streptomycin (GibcoBRL). Transient transfection was performed with lipofectamine 2000 (Invitrogen) following manufacturer's instructions.

\section{Manipulation of primary cells}

BMDCs were generated according to the culture method published previously [39]. BMDMs were obtained as described [29] with minor modifications. BMDM and BMDC were harvested after about 6-10 days and cultured in 12-well plates for siRNA transfection using lipofectamine 2000 or transfected directly with the nucleofactor kit (Amaxa) before culturing further. For peritoneal macrophages, 3 days after injection of $2 \mathrm{ml}$ of $4.0 \%$ (wt/vol) thioglycollate medium (Sigma), peritoneal cells were isolated from the peritoneal cavities of mice by peritoneal lavage with PBS. Macrophages were collected after washing twice with PBS and were resuspended in DMEM containing 10\% FBS in 12-well plates for further RNAi experiments.

\section{Sub-cellular fractionation}

HEK293 cells were washed with cold PBS and lysed by douncing in homogenization buffer (buffer $\mathrm{H}$ : $210 \mathrm{mM}$ sucrose, $70 \mathrm{mM}$ mannitol, $1 \mathrm{mM}$ EDTA, $1 \mathrm{mM}$ EGTA, $1.5 \mathrm{mM} \mathrm{MgCl}_{2}, 10 \mathrm{mM}$ Hepes (pH 7.2), protease inhibitor cocktail). The homogenate was centrifuged at $500 \times \mathrm{g}$ for $10 \mathrm{~min}$ and the pellet (P0) was saved as crude nuclei. The supernatant (S0) was centrifuged at $5000 \times g$ for $10 \mathrm{~min}$ to precipitate crude mitochondria (P5). The supernatant (S5) was further centrifuged at $50000 \times g$ for $10 \mathrm{~min}$ to generate S50 and $\mathrm{P} 50$. Each pellet was resuspended in buffer $\mathrm{H}$ for later experiments. 
Table 1 The primers used for Q-PCR

\begin{tabular}{|c|c|c|c|}
\hline \multicolumn{2}{|r|}{ Primers for human } & \multicolumn{2}{|r|}{ Primers for mouse } \\
\hline \multirow[t]{2}{*}{$\beta$-actin } & sense (5'-AAA GAC CTG TAC GCC AAC AC-3') & $\beta$-actin & sense (5'-AGA CCT CTA TGC CAA CAC AG-3') \\
\hline & antisense (5'-GTC ATA CTC CTG CTT GCT GAT-3') & & antisense (5'-TCG TAC TCC TGC TTG CTG AT-3') \\
\hline IFN- $\beta$ & antisense (5'-GGC CTT CAG GTA ATG CAG AA-3') & & antisense (5'-TCA GAA ACA CTG TCT GCT GG-3') \\
\hline ISG56 & sense (5'-GCC ATT TTC TTT GCT TCC CCT A-3') & ISG56 & sense (5'-AGT GCA GGC AGA AAT TCA CC-3') \\
\hline Rantes & antisense (5'-ACA CAC TTG GCG GTT CTT TC-3') & & antisense (5'-GTG ACA AAC ACG ACT GCA AG-3') \\
\hline \multirow[t]{2}{*}{ IL-8 } & sense (5'-AGG TGC AGT TTT GCC AAG GA-3') & IL-6 & sense (5'-GAG AGG AGA CTT CAC AGA GG-3') \\
\hline & antisense (5'-TTT CTG TGT TGG CGC AGT GT-3') & & antisense (5'-GTA CTC CAG AAG ACC AGA GG-3') \\
\hline A20 & sense (5'-GCG TTC AGG ACA CAG ACT TG-3') & IL-12p40 & sense (5'-GCT TCT TCA TCA GGG ACA TC-3') \\
\hline
\end{tabular}

Protein purification and mass spectrometry

At $36 \mathrm{~h}$ after transfection of Flag-MAVS, HEK293T cells were stimulated by $\mathrm{SeV}$ for $2 \mathrm{~h}$, and then collected and fractionated. The mitochondria pellet fraction was lysed with NP40 buffer (buffer $\mathrm{N}$ : $50 \mathrm{mM}$ Tris (pH 7.4), $150 \mathrm{mM} \mathrm{NaCl}, 1 \mathrm{mM} \mathrm{PMSF}, 0.1 \%$ (v/v) NP40, protease inhibitor cocktail). Post-centrifuged supernatants were pre-cleared with protein $\mathrm{A} / \mathrm{G}$ beads and control IgG for $0.5 \mathrm{~h}$ at $4{ }^{\circ} \mathrm{C}$. Pre-cleared lysates were mixed with $1-2 \mu \mathrm{g}$ of mouse antiFlag antibody for $1 \mathrm{~h}$ at $4{ }^{\circ} \mathrm{C}$. Then protein $\mathrm{A} / \mathrm{G}$ beads were added and the mixtures were incubated for $2 \mathrm{~h}$ at $4{ }^{\circ} \mathrm{C}$. Precipitates were washed extensively with Buffer N. Proteins bound to protein A/ $\mathrm{G}$ beads were separated on 7.5\% SDS-PAGE. After silver staining (Sigma), specific protein bands were excised and analyzed by iontrap mass spectrometry.

\section{Immunoblot analysis and immunoprecipitation assay}

For immunoblotting, the immunoprecipitates or whole-cell lysates were resolved by SDS-PAGE and transferred to a PVDF membrane (Millipore). Immunoblotting was probed with indicated antibodies. The proteins were visualized by using Supersignal West Pico chemiluminescence ECL kit (Pierce). For immunoprecipitation, cells were collected about 24-48 h after transfection and then lysed in Buffer $\mathrm{N}$ or sonicated in TBS buffer supplemented with a complete protease inhibitor cocktail (Roche). Then the whole-cell lysates were used for immunoprecipitation with indicated antibodies. Generally, 0.5-1 $\mu \mathrm{g}$ of commercial antibody was added to $500 \mu \mathrm{g}$ of cell lysate and then incubated for $1-2 \mathrm{~h}$ at $4{ }^{\circ} \mathrm{C}$. After addition of protein $\mathrm{A} / \mathrm{G}$ beads, the incubation was continued for 2-10 h. Immunoprecipitates were extensively washed with Buffer $\mathrm{N}$ and eluted with SDS loading buffer by boiling for $5 \mathrm{~min}$.

\section{GST pull-down assays}

For GST pull-down assay, $0.25-1 \mu \mathrm{g}$ of indicated recombinant proteins in buffer $\mathrm{N}$ were incubated with $15 \mu$ l of Glutathione Sepharose $4 \mathrm{~B}$ (GE Healthcare) for $1 \mathrm{~h}$ at $4{ }^{\circ} \mathrm{C}$. After washing three times with Buffer N, pellets were analyzed on SDS-PAGE and subjected to immunoblotting analysis.

\section{Confocal imaging}

HEK293T cells were plated on coverslips in 12-well plates and transfected with the indicated siRNA or plasmids. To label mitochondria in a specific experiment, cells were incubated with $250 \mathrm{nM}$ MitoTracker Red (Molecular Probes) for $30 \mathrm{~min}$ at $37^{\circ} \mathrm{C}$. Coverslips with the cells were washed once with PBS and fixed in $3.7 \%$ formaldehyde in PBS for $15 \mathrm{~min}$. Cells were permeabilized and blocked for $30 \mathrm{~min}$ at room temperature in a staining buffer containing Triton X-100 (0.1\%) and BSA (2\%), and then incubated with a primary antibody in the staining buffer lacking Triton $\mathrm{X}-100$ for $1 \mathrm{~h}$. After washing three times in the staining buffer lacking Triton X-100, cells were incubated with a secondary antibody for $1 \mathrm{~h}$ and then with DAPI for $10 \mathrm{~min}$. The coverslips were then washed extensively and fixed on slides. Imaging of the cells was carried out using Leica laser scanning confocal microscopy.

\section{Mitochondrial TMRM staining}

For stock solution, both CCCP (Sigma) and TMRM (Biotium) were dissolved in dimethylsulfoxide (DMSO; Sigma) to obtain $50 \mathrm{mM}$ concentration and stored at $-20{ }^{\circ} \mathrm{C}$. HEK293T cells were plated onto four Willco dishes; two dishes were transfected with the indicated siRNAs for 2 days; the other two dishes were treated with either CCCP or DMSO for $3 \mathrm{~h}$. All cells were co-transfected with EGFP-mito plasmids to label mitochondria. After incubating at $37{ }^{\circ} \mathrm{C}$ in the dark with $10 \mu \mathrm{M}$ TMRM for $30 \mathrm{~min}$, the cells were washed with DMEM (no phenol red) and imaged by Leica laser scanning confocal microscopy.

\section{Rescue experiments}

HEK293 cells were first treated with Tom70 or Hsp90 siRNA for $12 \mathrm{~h}$ and then transfected again with the indicated siRNAresistant Tom 70 or Hsp90 plasmids, followed by $\mathrm{SeV}$ infection the next day. 
Kinase assay

For each reaction, $500 \mu \mathrm{g}$ of cell lysate was incubated at $4{ }^{\circ} \mathrm{C}$ for $1 \mathrm{~h}$ with anti-TBK1 antibody $(4 \mu \mathrm{l})$ conjugated with protein $\mathrm{A} /$ $\mathrm{G}$ bead slurry $(10 \mu 1)$. Immunoprecipitates were washed twice with buffer $\mathrm{N}$ and three times with buffer K (20 mM HEPES ( $\mathrm{pH} 7.2$ ), $50 \mathrm{mM} \mathrm{NaCl}, 10 \mathrm{mM} \mathrm{MgCl}_{2}$ and $0.1 \mathrm{mM}$ EDTA). Then, the beads were incubated at $37^{\circ} \mathrm{C}$ for $30 \mathrm{~min}$ in $20 \mu \mathrm{l}$ of the buffer $\mathrm{K}$ containing $\gamma-{ }^{32} \mathrm{P}$-ATP $(5 \mu \mathrm{Ci})$, ATP $(100 \mu \mathrm{M})$ and GST-IRF3 $(1.0 \mu \mathrm{g})$. After SDS-PAGE, the phosphorylation of IRF3 was visualized by autoradiography.

\section{Measurement of IFN- $\beta$ production}

HEK293 cells were transfected with the indicated plasmids or siRNA, and then cell culture supernatants were collected at $8 \mathrm{~h}$ after virus infection and analyzed for IFN- $\beta$ production using ELISA kits (PBL Biomedical Laboratories) according to manufacturer's instructions.

\section{Virus manipulation}

Viral infection was performed when $80 \%$ cell confluence was reached. Then, culture media was replaced by serum-free DMEM, and $\mathrm{SeV}$, VSV or NDV-GFP was added to the media at MOI 0.2-1 according to specific experiments. After $1 \mathrm{~h}$, the medium was removed and the cells were fed with DMEM containing 10\% FBS.

\section{Statistics}

Student's $t$-test was used for the comparison of two independent treatments. For all tests, a $P$ value $<0.05$ was considered statistically significant.

\section{Acknowledgment}

This work was supported by grants from the Ministry of Science and Technology of Shanghai (09XD1404800); National Natural Science Foundation of China (30623003, 30225013); Ministry of Science and Technology of China (2006CB504301, 2007CB914504, 2010CB912026); and Chinese Academy of Sciences (KSCX1-YW-R-06). We thank Drs Lin Li (SIBS), Jiansheng Kang (SIBS), Quan Chen (Nankai University), Mian Wu (University of Science and Technology of China) and Hongbing Shu (Wuhan University) for their technical assistance in this study.

\section{References}

1 Stetson DB, Medzhitov R. Type I interferons in host defense. Immunity 2006; 25:373-381.

2 Uematsu S, Akira S. Toll-like receptors and type I interferons. J Biol Chem 2007; 282:15319-15323.

3 Yonheyama M, Kikuchi M, Natsukawa T, et al. The RNA helicase RIG-I has an essential function in double-stranded RNA-induced innate antiviral responses. Nat Immunol 2004; 5:730-737.

4 Yoneyama M, Kikuchi M, Matsumoto K, et al. Shared and unique functions of the DExD/H-box helicases RIG-I, MDA5, and LGP2 in antiviral innate immunity. J Immunol 2005; 175:2851-2858.

5 Gitlin L, Barchet W, Gilfillan S, et al. Essential role of Mda-5 in type I IFN responses to polyriboinosinic:polyribocytidylic acid and encephalomyocarditis picornavirus. Proc Natl Acad Sci USA 2006; 103:8459-8464.

6 Kato H, Takeuchi O, Sato S, et al. Differential roles of MDA5 and RIG-I helicases in the recognition of RNA viruses. Nature 2006; 441:101-105.

7 Pichlmair A, Schulz O, Tan CP, et al. RIG-I-mediated antiviral responses to single-stranded RNA bearing 5'-phosphates. Science 2006; 314:997-1001.

8 Takeuchi O, Akira S. MDA5/RIG-I and virus recognition. Curr Opin Immunol 2008; 20:17-22.

9 Kawai T, Takahashi K, Sato S, et al. IPS-1, an adaptor triggering RIG-I- and Mda5-mediated type I interferon induction. Nat Immunol 2005; 6:981-988.

10 Meylan E, Curran J, Hofmann K, et al. Cardif is an adaptor protein in the RIG-I antiviral pathway and is targeted by hepatitis C virus. Nature 2005; 437:1167-1172.

11 Seth RB, Sun L, Ea CK, Chen ZJ. Identification and characterization of MAVS, a mitochondrial antiviral signaling protein that activates NF-kappaB and IRF 3. Cell 2005; 122:669-682.

$12 \mathrm{Xu} \mathrm{LG}$, Wang YY, Han KJ, et al. VISA is an adapter protein required for virus-triggered IFN-beta signaling. Molecular Cell 2005; 19:727-740.

13 Michallet MC, Meylan E, Ermolaeva MA, et al. TRADD protein is an essential component of the RIG-like helicase antiviral pathway. Immunity 2008; 28:651-661.

14 Fitzgerald KA, McWhirter SM, Faia KL, et al. IKKepsilon and TBK1 are essential components of the IRF3 signaling pathway. Nat Immunol 2003; 4:491-496.

15 Sharma S, tenOever BR, Grandvaux N, et al. Triggering the interferon antiviral response through an IKK-related pathway. Science 2003; 300:1148-1151.

16 McWhirter SM, Fitzgerald KA, Rosains J, et al. IFN-regulatory factor 3-dependent gene expression is defective in Tbk1deficient mouse embryonic fibroblasts. Proc Natl Acad Sci USA 2004; 101:233-238.

17 Lin R, Heylbroeck C, Pitha PM, Hiscott J. Virus-dependent phosphorylation of the IRF-3 transcription factor regulates nuclear translocation, transactivation potential, and proteasome-mediated degradation. Mol Cell Biol 1998; 18:2986-2996.

18 Kumar KP, McBride KM, Weaver BK, Dingwall C, Reich NC. Regulated nuclear-cytoplasmic localization of interferon regulatory factor 3, a subunit of double-stranded RNA-activated factor 1. Mol Cell Biol 2000; 20:4159-4168.

19 Sato M, Suemori H, Hata N, et al. Distinct and essential roles of transcription factors IRF-3 and IRF-7 in response to viruses for IFN-alpha/beta gene induction. Immunity 2000; 13:539548.

20 Lin R, Lacoste J, Nakhaei P, et al. Dissociation of a MAVS/ IPS-1/VISA/Cardif-IKKepsilon molecular complex from the mitochondrial outer membrane by hepatitis C virus NS3-4A proteolytic cleavage. $J$ Virol 2006; 80:6072-6083.

21 Paz S, Vilasco M, Arguello M, et al. Ubiquitin-regulated recruitment of IkappaB kinase epsilon to the MAVS interferon signaling adapter. Mol Cell Biol 2009; 29:3401-3412.

22 Zeng W, Xu M, Liu S, Sun L, Chen ZJ. Key role of Ubc5 and lysine-63 polyubiquitination in viral activation of IRF3. Mol Cell 2009; 36:315-325.

23 Baker MJ, Frazier AE, Gulbis JM, Ryan MT. Mitochondrial protein-import machinery: correlating structure with function. 
Trends Cell Biol 2007; 17:456-464.

24 Neupert W, Herrmann JM. Translocation of proteins into mitochondria. Annu Rev Biochem 2007; 76:723-749.

25 Young JC, Hoogenraad NJ, Hartl FU. Molecular chaperones Hsp90 and Hsp70 deliver preproteins to the mitochondrial import receptor Tom70. Cell 2003; 112:41-50.

$26 \mathrm{Wu}$ Y, Sha B. Crystal structure of yeast mitochondrial outer membrane translocon member Tom70p. Nat Struct Mol Biol 2006; 13:589-593.

27 Abe Y, Shodai T, Muto T, et al. Structural basis of presequence recognition by the mitochondrial protein import receptor Tom20. Cell 2000; 100:551-560.

28 Yang K, Shi H, Qi R, et al. Hsp90 regulates activation of interferon regulatory factor 3 and TBK-1 stabilization in Sendai virus-infected cells. Mol Biol Cell 2006; 17:1461-1471.

29 Sun Q, Sun L, Liu HH, et al. The specific and essential role of MAVS in antiviral innate immune responses. Immunity 2006; 24:633-642.

30 Ishikawa H, Barber GN. STING is an endoplasmic reticulum adaptor that facilitates innate immune signalling. Nature 2008; 455:674-678.

31 Zhong B, Yang Y, Li S, et al. The adaptor protein MITA links virus-sensing receptors to IRF3 transcription factor activation. Immunity 2008; 29:538-550.

32 Poeck H, Bscheider M, Gross O, et al. Recognition of RNA virus by RIG-I results in activation of CARD9 and inflammasome signaling for interleukin 1 beta production. Nature immunology 2010; 11:63-69.

33 Tang ED, Wang CY. MAVS self-association mediates antiviral innate immune signaling. J Virol 2009; 83:3420-3428.

34 Kayagaki N, Phung Q, Chan S, et al. DUBA: a deubiquitinase that regulates type I interferon production. Science 2007; 318:1628-1632.

35 You F, Sun H, Zhou X, et al. PCBP2 mediates degradation of the adaptor MAVS via the HECT ubiquitin ligase AIP4. Nat Immunol 2009; 10:1300-1308.

36 Kawagoe T, Takeuchi O, Takabatake Y, et al. TANK is a negative regulator of Toll-like receptor signaling and is critical for the prevention of autoimmune nephritis. Nat Immunol 2009; 10:965-72.

37 Chen G, Cao P, Goeddel DV. TNF-induced recruitment and activation of the IKK complex require $\mathrm{Cdc} 37$ and Hsp90. Molecular Cell 2002; 9:401-410.

38 Moore CB, Bergstralh DT, Duncan JA, et al. NLRX1 is a regulator of mitochondrial antiviral immunity. Nature 2008; 451:573-577.

39 Lutz MB, Kukutsch N, Ogilvie AL, et al. An advanced culture method for generating large quantities of highly pure dendritic cells from mouse bone marrow. J Immunol Methods 1999; 223:77-92.

(Supplementary information is linked to the online version of the paper on Cell Research website.) 